ALEA, Lat. Am. J. Probab. Math. Stat. 18, 349-377 (2021)

DOI: $10.30757 /$ ALEA.v18-16

\title{
Counting excursions: symmetries, knock-ins and non-linear formula for Itô-McKean diffusions
}

\author{
Maciej Wiśniewolski \\ Institute of Mathematics, University of Warsaw, Banacha 2, 02-097 Warszawa, Poland \\ E-mail address: wisniewolski@mimuw.edu.pl
}

\begin{abstract}
Excursion theory is revisited on the ground of Itô-McKean diffusions. There are raised questions about symmetries, knock-in processes, excursion local time and the non-linear version of the master formula of excursions. The questions are answered due to introducing the counting excursion technique. The technique is a synthesis of straddling at time approach, the classical, potential in spirit approach, and the theory of convolution algebra of locally integrable functions, generalized later in this work for the convolutions of $\sigma$-finite measures. Some examples are presented, including the famous problem of expressing the density of first hitting time of Ornstein-Uhlenbeck process in terms of elementary functions.
\end{abstract}

\section{Introduction}

On the ground of classical theory of Itô-McKean diffusions we raise questions related to excursion theory of Markov processes. These concern symmetries, knock-in processes, excursion local time and finally the non-linear version of master formula of excursions. The primal reference for the theory of Itô-McKean diffusions (called later in this paper simply diffusions) is the monograph Itô and McKean (1974). The references for excursion theory are again Itô and McKean (1974) but also Rogers and Williams (1987) or Revuz and Yor (1991). Excursion theory, initiated originally by Itô (1972), is a probabilistic method of describing the paths of Markov processes by setting their fragments into a Poisson structured space. The synthesis of several concepts of excursion theory is the Blumenthal's acclaimed book (Blumenthal, 1992).

Our contribution to excursion theory for diffusions relies on developing the new computational technique, called later counting excursions. We divide a path of a diffusion $X$ into the excursions from a level $x$ and for a fixed time $t>0$ we consider some integral functionals of $X$ on $[0, t]$. We count the excursions before $t$ and

Received by the editors August 25th, 2019; accepted December 10th, 2020.

2010 Mathematics Subject Classification. 60J35, 60J60, 60J55.

Key words and phrases. linear diffusion, local time, excursions of Markov processes, counting excursions technique, convolutions of locally integrable functions and measures. 
complement the functional by the fragment straddling at $t$. The regular excursion fragments are described by the master formula in terms of excursion measure and an associated local time. The complemented fragment straddling at $t$ is described by means of potential theory. Our technique is to compare obtained description with the information coming from the distribution of $X$ and its transition density. Some elements of the technique have been already used for studies on Bessel processes with negative index in Byczkowski et al. (2019). For the straddling at time approach see the comprehensive study of Getoor (1979).

The reason for the choice of the diffusive setup does not come from the necessity to work on a process with continuous paths, but rather from the fact that Itô-McKean diffusions are regular and rich with useful objects for the effective distributional characterization. For a detailed discussion on such a setup and its use in excursion theory see the monograph of Salminen, Vallois and Yor (Salminen et al., 2007). Our contribution to the theory of Itô-McKean diffusions relies on using the algebra of convolution of locally integrable functions on non-negative half-line. Combining the counting excursions technique with convolution algebra approach turns out to be essential for answering the questions raised above. A recent study on convolution algebra approach for the notions of first hitting time, local time and generalized Stroock Wiliams equation can be also found in Jakubowski and Wiśniewolski (2020) and Jakubowski and Wiśniewolski (2020+).

In what follows we give an overview of our results. For a diffusion $X$ and points $x, b$, we consider excursions from $x$ and a killed diffusion obtained by sending $X$ to the cemetery state at the first hitting time of $b$. We develop a new formula joining the transition densities of a killed processes and the corresponding first hitting time. Joining it with the formula of Hunt, we will discover symmetries of transition densities of killed processes and new relations between densities of first hitting times and the Lévy measure of inverse local time of $X$ at $x$. It is well known that the distribution of the first hitting time may be asymmetric in a sense that there is an elementary formula for the density of hitting $x$ when one starts from $b$, and at the same time we do not know the accessible formula for the case, when the roles of $b$ and $x$ are exchanged. We present a new representation of the density of the first hitting time of $b$ when $X$ starts from $x$ in terms of the following objects: transition density of $X$, the Lévy measure of inverse local time at $x$ and finally, the first hitting time of $x$ when the process starts from $b$. We present a new approach to well known problem of finding the formula of the density of the first hitting time of $b$ by an Ornstein-Uhlenbeck process which starts from 0 (see Alili et al., 2005, Lipton and Kaushansky, 2018, Hamana, 2020).

For the two fixed regular points of the state space (say $x$ and $b$ ) we study symmetries for additive functionals of $X$. We present several dependencies between expectations of functionals considered under excursion measures $\widehat{\mathbb{P}}_{x}$ and $\widehat{\mathbb{P}}_{b}$ respectively. We also define the double-knock-in process which behaves like $X$ until it exits the given interval $I$. Then it becomes the process killed at $x$. The knock-in process is defined analogously. We develop formula joining the transition densities of the process killed at $x$ and the process killed at the exit from $I$. This leads to a conclusion that the transition density of a double-knock-in is the convolution of the density of the process killed at first exit from $I$ and the density of first hitting time of a single point. Moreover, using these concepts we generalize the classical Hunt formula. The notion of knock-in constructions is strictly connected with the 
financial problem of pricing and hedging so called barrier options (see Mijatović and Pistorius, 2013).

Next we study the distribution of excursion local time. The concept of excursion local time for a Brownian motion is presented in Trotter (1958) and in a more general context in Blumenthal (1992, Ch. VI). A brilliant application of the notion of excursion local time for branching processes was presented by Le Gall (1991). We find the convolution formula for the Laplace transform of excursion local time. For this, inspired mostly by the work of Lew (1972), we develop the theory of convolutions of $\sigma$-finite measures.

Last but not least we present a generalization of the master formula of excursions for the non-linear case. Although the exponential version of the master formula for additive functionals of a Markov process $X$ has been known before (see Revuz and Yor, 1991, Ch. XII, Sect. 2), it has not built any deeper understanding of the non-linear case of the excursion formula. We shed a new light on the the last problem. We find a non-linear formula for the class of additive functionals. The structure of our result reminds the Getoor's description (Getoor, 1979) of straddling at time excursions, but unlike their formula our result has not a local character. We present a Markovian kernel describing the sum of excursions under the arbitrary chosen function. The presentation of non-linear version of the master formula in terms of the Markovian kernel is exactly in the spirit of general excursion theory (see Blumenthal, 1992, Ch. VII).

\section{Preliminaries}

On a complete probability space $(\Omega, \mathcal{F}, \mathbb{P})$ endowed with a filtration $\left(\mathcal{F}_{t}\right)_{t \in[0, \infty)}$ satisfying the usual conditions we consider a diffusion $X$ with the state space $E=$ $(l, r) \subset \mathbb{R}$ where $l$ and $r$ do not need to be finite. The death-time of $X$ is denoted by $\zeta$ and we assume that $X$ is continuous on $[0, \zeta)$. Not loosing much generality we will always assume that $\zeta=\infty$ (see discussion in Getoor (1979)). We assume that each point $y \in E$ is a regular point. We use standard notation for the measure $\mathbb{P}_{x}$ under which $X_{0}=x$ a.s. By $\theta$ we denote the shift operator. We also assume that for any $x \in \operatorname{Int}(E)$ and $y \in E$ we have $\mathbb{P}_{x}\left(\sigma_{y}<\infty\right)>0$, where $\sigma_{y}$ denotes the first hitting time of $y$.

A linear diffusion has three basic characteristics defined on $E$ : the speed measure $m$, a scale function $s$ and the killing measure $k$, whose properties determine the behavior of $X$ in the vicinity of the endpoints of $E$. In general, the functions $m, s, k$ are responsible for boundary classification (see, e.g., Itô and McKean, 1974, pp.108 and Borodin and Salminen, 2002, Chapter II, Section 1). We will always assume that $k \equiv 0$.

It is well known (see Salminen et al., 2007, 1.2 or Borodin and Salminen, 2002, Chapter II, pp. 4) that in the above setup a diffusion $X$ has a jointly continuous transition density $p_{t}(x, y)$ with respect to the speed measure $m$, i.e.

$$
\mathbb{P}_{x}\left(X_{t} \in A\right)=\int_{A} p_{t}(x, y) m(d y)
$$

for every Borel subset $A$ of $E$. Moreover, $p$ is symmetric, that is, $p_{t}(x, y)=p_{t}(y, x)$. The Green (resolvent) kernel of $X$ is defined for $\lambda>0$ by

$$
R_{\lambda}(x, y)=\int_{0}^{\infty} e^{-\lambda t} p_{t}(x, y) d t
$$


The last object is especially useful for the description of the Laplace transform of $\sigma_{y}$, since

$$
\mathbb{E}_{x} e^{-\lambda \sigma_{y}}=\frac{R_{\lambda}(x, y)}{R_{\lambda}(y, y)}
$$

(see Salminen et al., 2007, formula (3)). It is known that $\sigma_{y}$ has under $\mathbb{P}_{x}$ a density $g_{y}^{x}$, and moreover the mapping $t \mapsto g_{y}^{x}(t)$ is continuous as follows from the eigendifferential expansions and discussion in Itô and McKean (1974, pp. 153 and 217) (see also Kent, 1980). By $\left(L^{x}\right)$ we denote the jointly continuous version the local time of $X$ at the point $x$, which is well defined due to assumptions on regularity of points from $E$. We assume that the local time is normalized such that for every measurable $f$ on $E$ and $t>0$

$$
\int_{0}^{t} f\left(X_{s}\right) d s=\int_{E} f(z) L_{t}^{z} m(d z) \quad \text { a.s. }
$$

We will also need some elements of convolution algebra theory. In particular for locally integrable $f, g$ we denote by $f * g$ their convolution

$$
(f * g)(t)=\int_{0}^{t} f(u) g(t-u) d u, \quad t \geq 0 .
$$

The background of the theory of convolution algebra will be given in Section 3 .

We assume that our probability space is a canonical space and that the coordinate process $X$ is a linear diffusion under a measure $\mathbb{P}_{x}$. The setup here is standard and $\Omega$ is a set of continuous $E$-valued functions with associated $\sigma$-field of measurable functions and filtration $\left(\mathcal{F}_{t}\right)_{t \in[0, \infty)}$ satisfying usual conditions. We assume that the speed measure $m$ does not have atoms. It is well known that for a linear diffusion, due to existence of the local time at $x \in E$, the classical Itô theory of excursions of the process $X$ from the point 0 may be applied (see Blumenthal, 1992, Chapter III). Let $U$ be the subspace of continuous functions $u:[0, \infty) \rightarrow E$ such that $0<R(u) \leq \infty$, where $R(u)=\inf \{t>0: u(t)=x\}$ and $u(0)=x, u(v)>0$ for $v \in(0, R(u))$. Moreover, we demand that $u(t)=x$ for all $t \geq R(u)$. Let $\mathcal{U}$ be the $\sigma$-algebra generated by the coordinate mappings. Let $\delta:[0, \infty) \rightarrow E$ be a constant function, $\delta(t) \equiv x$. We denote by $U^{\delta}$ the space $U \cup\{\delta\}$, and set $\mathcal{U}^{\delta}=\sigma(U,\{\delta\})$ (actually $\delta$ serves only for the formal definition of excursion path-space and will not be used in the sequel). Finally, on the canonical space we consider an excursion point process $\left(e_{s}\right)_{s>0}$ and we denote by $\widehat{\mathbb{P}}_{x}(\cdot)$ the associated measure of excursions from $x$ (see Blumenthal, 1992, Chapter III). If a diffusion is recurrent then the point process $\left(e_{s}\right)_{s>0}$ is a Poisson point process and the theory is built upon the stochastic integral with respect to Poisson process (see Revuz and Yor, 1991, Section 1 Chapter XII). However, in the general case the theory is build on the properties of excessive functions and in particular on the existence of the associated additive functional, which in case of excursions from the point is simply a local time. One should notice that the existence of excursion measure, fundamental master formula and Markov property under the measure of excursions in no way depend on the recurrence hypothesis (see Blumenthal, 1992, Section 3, point (g), Chapter III). Thus we are not limited by the recurrence assumption.

The path-space of excursions can be seen as an immersion into the canonical space. If the measure of excursions from $x$ is denoted by $\widehat{\mathbb{P}}_{x}$, we recall the well known fact that a coordinate process $X$ killed at $R$ (denoted by $\widehat{X}$ ) is under $\widehat{\mathbb{P}}_{x}$ a Markov process with a transition function equal to the transition function of the 
initial diffusion killed at $x$. The advantage of working under the diffusive setup is that the entrance law of $\widehat{X}$ is given on measurable subsets of $E \backslash\{x\}$ by

$$
\widehat{\mathbb{P}}_{x}\left(\widehat{X}_{t} \in d z\right)=g_{x}^{z}(t) m(d z)
$$

(see Salminen et al., 2007, Sect. 2).

\section{Symmetries for the transition densities of killed diffusions}

In what follows we assume that a diffusion $\widehat{X}^{(b)}$, built on $X$ by killing at first hitting time of $b \in E$, has a transition density with respect to the speed measure, denoted by $\widehat{p}^{(b)}$. Although we will regularly omit the dependence of $\widehat{p}^{(b)}$ on $t$, we always have in mind that

$$
\widehat{p}_{t}^{(b)}(x, z) m(d z)=\mathbb{P}_{x}\left(\widehat{X}_{t}^{(b)} \in d z\right), \quad t>0 .
$$

In the sequel we will use the classical result of Hunt saying that

$$
\widehat{p}^{(b)}(x, z)=p(x, z)-p(b, z) * g_{b}^{x},
$$

(see Bogus and Małecki, 2015). The notation of a killed diffusion should not be confused with a coordinate process under excursion measure. The following new representation of $\widehat{p}^{(b)}$ is a consequence of application of the counting excursions technique.

Theorem 3.1. Assume that $x, z, b \in E$. Then

$$
\widehat{p}^{(b)}(x, z)=g_{x}^{z} * \widehat{p}^{(b)}(x, x)-g_{b}^{x} * \widehat{p}^{(x)}(b, z) .
$$

Proof: Let $\left(e_{s}\right)_{s>0}$ be the point process of excursions away from the set $\{x\}$ of the process $X$, and $\widehat{\mathbb{P}}_{x}(\cdot)$ be the corresponding excursion measure on an associated path space. Assume that $t>0$ is fixed, $\sigma_{b}$ is the first hitting time of $b$, and $f$ is a Borel positive or bounded function $f$ on $E$. We write the integral $\mathbb{E}_{x} \int_{0}^{t \wedge \sigma_{b}} e^{-\lambda r} f\left(X_{r}\right) d r$ in terms of excursions $\left(e_{s}\right)$. For this let $d_{t}^{x}:=\inf \left\{s \geq t: X_{s}=x\right\}$, and for $\left(L^{x}\right)$ the process of local time of $X$ at $x$, let

$$
\tau_{t}^{x}=\inf \left\{s>0: L_{s}^{x}>t\right\}, \quad \tau_{t-}^{x}=\inf \left\{s>0: L_{s}^{x} \geq t\right\} .
$$

By $G$ we denote the set of left hand sides of excursion intervals, that is

$$
G=\left\{\tau_{r-}^{x}: \tau_{r}^{x}>\tau_{r-}^{x}\right\} .
$$

Since we assumed that the speed measure $m$ does not have atoms, and thus there is no mass put on the set $\{x\}$, we can write for a Borel positive and bounded function $f$ on $E$

$\mathbb{E}_{x} \int_{0}^{t \wedge \sigma_{b}} e^{-\lambda r} f\left(X_{r}\right) d r=\mathbb{E}_{x} \sum_{G \cap\left[0, t \wedge \sigma_{b}\right)} \int_{\tau_{r-}^{x}}^{\tau_{r}^{x}} e^{-\lambda s} f\left(X_{s}\right) d s-\mathbb{E}_{x}\left[\int_{t \wedge \sigma_{b}}^{d_{t \wedge \sigma_{b}}^{x}} e^{-\lambda s} f\left(X_{s}\right) d s\right]$

$$
=I(t)-I I(t) .
$$

Observe that

$I(t)=\mathbb{E}_{x} \sum_{G \cap\left[0, t \wedge \sigma_{b}\right)} e^{-\lambda \tau_{r-}^{x}}\left(\int_{0}^{\sigma_{x}} e^{-\lambda s} f\left(X_{s}\right) d s\right) \circ \theta_{\tau_{r-}^{x}}=\mathbb{E}_{x} \sum_{\left\{r: \tau_{r-}^{x}<t \wedge \sigma_{b}\right\}} e^{-\lambda \tau_{r-}^{x}} F_{f}\left(e_{r}\right)$, 
where $F(u)=\int_{0}^{R(u)} e^{-\lambda s} f(u(s)) d s$. The master formula of excursions (see, e.g., Blumenthal, 1992, Theorem 3.24 and formula (3.27) in Chapter III) applied to the excursion point process $\left(e_{s}\right)$, parametrized in line with Itô's theory by the process of inverse local time at 0 , yields

$$
\begin{aligned}
\mathbb{E}_{x} \sum_{\left\{r: \tau_{r-}^{x}<t \wedge \sigma_{b}\right\}} e^{-\lambda \tau_{r-}^{x}} F_{f}\left(e_{r}\right) & =\mathbb{E}_{x} \int_{0}^{t \wedge \sigma_{b}} e^{-\lambda s}\left[\int_{U} F_{f}(u) \widehat{\mathbb{P}}_{x}(d u)\right] d L_{s}^{x} \\
& =\int_{U} F_{f}(u) \widehat{\mathbb{P}}_{x}(d u)\left(\mathbb{E}_{x} \int_{0}^{t} e^{-\lambda s} d L_{s \wedge \sigma_{b}}^{x}\right) .
\end{aligned}
$$

On the other hand, by strong Markov property

$$
I I(t)=\mathbb{E}_{x}\left[\int_{t \wedge \sigma_{b}}^{d_{t \wedge \sigma_{b}}^{x}} e^{-\lambda s} f\left(X_{s}\right) d s\right]=\mathbb{E}_{x} e^{-\lambda t \wedge \sigma_{b}} \mathbb{E}_{X_{t \wedge \sigma_{b}}}\left[\int_{0}^{\sigma_{x}} e^{-\lambda s} f\left(X_{s}\right) d s\right] .
$$

Thus, letting now $t \rightarrow \infty$ in (3.3) and some standard limit arguments yield

$$
\begin{aligned}
\mathbb{E}_{x} \int_{0}^{\sigma_{b}} e^{-\lambda r} f\left(X_{r}\right) d r=\int_{U} F_{f}(u) \widehat{\mathbb{P}}_{x}(d u)\left(\mathbb{E}_{x} \int_{0}^{\infty} e^{-\lambda s} d L_{s \wedge \sigma_{b}}^{x}\right) \\
-\mathbb{E}_{x} e^{-\lambda \sigma_{b}}\left[\mathbb{E}_{b} \int_{0}^{\sigma_{x}} e^{-\lambda s} f\left(X_{s}\right) d s\right] .
\end{aligned}
$$

We have

$$
\mathbb{E}_{x} \int_{0}^{\sigma_{b}} e^{-\lambda r} f\left(X_{r}\right) d r=\int_{E} f(z) \int_{0}^{\infty} e^{-\lambda s} \widehat{p}_{s}^{(b)}(x, z) d s m(d z) .
$$

Define a measure on $\mathcal{B}\left(\mathbb{R}_{+}\right)$by $\mu_{x, b}(0, t):=\mathbb{E}_{x} L_{t \wedge \sigma_{b}}^{x}$, so that

$$
\mathbb{E}_{x} \int_{0}^{\infty} e^{-\lambda s} d L_{s \wedge \sigma_{b}}^{x}=\int_{0}^{\infty} e^{-\lambda s} \mu_{x, b}(d s) .
$$

Integration by parts yields easily that

$$
\int_{0}^{\infty} e^{-\lambda s} \mu_{x, b}(d s)=\lambda \int_{0}^{\infty} e^{-\lambda s} \mu_{x, b}(0, s) d s .
$$

By immersion, $u(\cdot)$ on $(0, R(u))$ can be identified with the coordinate process $\widehat{X}$ killed at $R$, which is under $\widehat{\mathbb{P}}$ a Markov process with the entrance law given by $\widehat{\mathbb{P}}\left(X_{t} \in d z\right)=g_{x}^{z}(t) m(d z)$ and the semigroup of the initial diffusion killed at the first hitting time of $x$. Thus, recalling the definition of $F_{f}$ we obtain

$$
\int_{U} F_{f}(u) \widehat{\mathbb{P}}_{x}(d u)=\int_{E \backslash\{x\}} \int_{0}^{\infty} e^{-\lambda s} f(z) g_{x}^{z}(s) d s m(d z) .
$$

By Fubini's theorem and a property of convolution

$$
\begin{aligned}
\mathbb{E}_{x} e^{-\lambda \sigma_{b}} & \left(\mathbb{E}_{b} \int_{0}^{\sigma_{x}} e^{-\lambda s} f\left(X_{s}\right) d s\right) \\
& =\left(\int_{0}^{\infty} e^{-\lambda s} g_{b}^{x}(s) d s\right)\left(\int_{0}^{\infty} \int_{E} e^{-\lambda s} f(z) \widehat{p}_{s}^{(x)}(b, z) m(d z) d s\right) \\
& =\int_{E} \int_{0}^{\infty} e^{-\lambda s} f(z)\left(g_{b}^{x} * \widehat{p}^{(x)}(b, z)\right)(s) d s m(d z) .
\end{aligned}
$$


Inserting just obtained identities into (3.4), using the fact that $m$ does not charge a single point and arbitrary choices of $\lambda$ and $f$ yield a.e. that for $t>0, z \in E$

$$
\widehat{p}_{t}^{(b)}(x, z)=\int_{0}^{t} g_{x}^{z}(t-s) \mu_{x, b}(d s)-\int_{0}^{t} g_{b}^{x}(t-s) \widehat{p}_{s}^{(x)}(b, z) d s .
$$

Let $\bar{\mu}_{x, b}(t):=\mu_{x, b}(0, t)$. Using (3.5) we may rewrite the last equality as

$$
1 * \widehat{p}^{(b)}(x, z)=g_{x}^{z} * \bar{\mu}_{x, b}-1 * g_{b}^{x} * \widehat{p}^{(x)}(b, z) .
$$

A simple use of occupation time formula shows that $\bar{\mu}_{x, b}=1 * \widehat{p}^{(b)}(x, x)$. Indeed, the last follows from identity

$$
\mathbb{E}_{x} \int_{0}^{t \wedge \sigma_{b}} f\left(X_{s}\right) d s=\mathbb{E}_{x} \int_{E} f(z) L_{t \wedge \sigma_{b}}^{z} m(d z),
$$

which holds for arbitrary Borel positive bounded $f$. As a result we rewrite (3.6) in the form

$$
1 * \widehat{p}^{(b)}(x, z)=g_{x}^{z} * 1 * \widehat{p}^{(b)}(x, x)-1 * g_{b}^{x} * \widehat{p}^{(x)}(b, z),
$$

so using the fact that the algebra of locally integrable function on positive half-line, with operation of convolution has not 0-divisors, we finally obtain

$$
\widehat{p}^{(b)}(x, z)=g_{x}^{z} * \widehat{p}^{(b)}(x, x)-g_{b}^{x} * \widehat{p}^{(x)}(b, z) .
$$

As a simple corollary of the last theorem we obtain the symmetric formulas for transition densities of killed diffusions.

Corollary 3.2. For $x, b \in E$ we have

$$
\begin{aligned}
g_{x}^{b} * \widehat{p}^{(b)}(x, x) & =g_{b}^{x} * \widehat{p}^{(x)}(b, b), \\
p(b, b) * \widehat{p}^{(b)}(x, x) & =p(x, x) * \widehat{p}^{(x)}(b, b) .
\end{aligned}
$$

Proof: Recalling that $(x, z) \mapsto p(x, z)$ is jointly continuous, we may let $z$ tend to $b$ and conclude from the Hunt formula (3.1) that $\lim _{z \rightarrow b} \widehat{p}^{(b)}(x, z)=0$. Now observe, that formula (3.2) and the Hunt formula imply that $z \mapsto g_{x}^{z} * \widehat{p}^{(b)}(x, x)$ is continuous. Moreover

$$
0=\lim _{z \rightarrow b}\left(g_{x}^{z} * \widehat{p}^{(b)}(x, x)-g_{b}^{x} * \widehat{p}^{(x)}(b, z)\right)=g_{x}^{b} * \widehat{p}^{(b)}(x, x)-g_{b}^{x} * \widehat{p}^{(x)}(b, b) .
$$

The first assertion follows. To obtain the second formula, we rewrite (3.7) in the form

$$
p(b, b) * p(x, x) * g_{x}^{b} * \widehat{p}^{(b)}(x, x)=p(b, b) * p(x, x) * g_{b}^{x} * \widehat{p}^{(x)}(b, b),
$$

repeat the identity $p(y, y) * g_{y}^{x}=p(x, y)$ which holds for any $x, y \in E$, and use the fact the there are no 0 -divisors in the convolution algebra of locally integrable functions on a positive half-line.

Recall that by $\widehat{\mathbb{P}}_{x}$ we denoted the measure of excursions of $X$ from the fixed point $x \in E$, and by $R$ we denoted the life-time of an excursion. Define a measure $\gamma_{x}(d t)=\widehat{\mathbb{P}}_{x}(R \in d t)$. It is well known that $\gamma_{x}$ is a Lévy measure of the inverse of 
local time of $X$ at $x$ denoted by $\left(\tau_{t}^{x}\right)_{t \geq 0}$. For a locally integrable $f$ by $f * \gamma_{x}$ we denote the convolution (with respect to a $\sigma$-finite measure)

$$
\left(f * \gamma_{x}\right)(t)=\int_{0}^{t} f(t-u) \gamma_{x}(d u)
$$

If $f$ is an element of $\mathcal{A}$ and $\mu$ is a $\sigma$-finite signed measure on $\mathcal{B}\left(\mathbb{R}_{+}\right)$, we may define $(f * \mu)(t)=\int_{0}^{t} f(t-u) \mu(d u)$, and since $f * \mu \in \mathcal{A}$ we may continue with recursive definition setting $f * \mu^{*(n+1)}:=\left(f * \mu^{* n}\right) * \mu, n \geq 1$. The last definition will play an important role in Section 5.

Corollary 3.3. Assume that $x, b \in E$. We have

$$
g_{b}^{x}+g_{b}^{x} * g_{x}^{b}=\widehat{p}^{(b)}(x, x) * \gamma_{x} .
$$

Proof: We use formula (3.2) to write

$$
\begin{aligned}
\int_{E} \widehat{p}_{t}^{(b)}(x, z) m(d z) & =\int_{E} \int_{0}^{t} g_{x}^{z}(u) \widehat{p}_{t-u}^{(b)}(x, x) \operatorname{dum}(d z) \\
& -\int_{E} \int_{0}^{t} g_{b}^{x}(t-u) \widehat{p}_{u}^{(x)}(b, z) \operatorname{dum}(d z) .
\end{aligned}
$$

By the definition of a killed process, Fubini's theorem, the definition of entrance law of coordinate process under $\widehat{\mathbb{P}}_{x}$ (that is by formula (2.3)) and the last equality we obtain

$$
\mathbb{P}_{x}\left(\sigma_{b}>t\right)+\int_{0}^{t} g_{b}^{x}(t-u) \mathbb{P}_{b}\left(\sigma_{x}>u\right) d u=\int_{0}^{t} \widehat{p}_{t-u}^{(b)}(x, x) \widehat{\mathbb{P}}_{x}(R>u) d u .
$$

Hence

$$
g_{b}^{x}+g_{b}^{x} * g_{x}^{b}=\widehat{p}^{(b)}(x, x) * \gamma_{x}
$$

as asserted.

In some cases, the use of some clever analytic and probabilistic arguments may result in the explicit form of $g_{x}^{b}$ for fixed $x, b \in E$. At the same time, we may fail to establish the analogous simple formula for $g_{b}^{x}$. To give an example of asymmetry of this kind, recall the famous case of Ornstein-Uhnlenbeck process where the form of $g_{0}^{b}$ is well known and relatively simple, while the form of $g_{b}^{0}$ appears only as a series of complicated functions or complicated integral transforms (see Alili et al., 2005 for a detailed discussion). In what follows we will find the form of functional $H$ such that (writing informally)

$$
g_{b}^{x}=H \cdot\left(p(x, x), \gamma_{x}(\cdot), g_{x}^{b}\right) .
$$

We will then present how the last works for Ornstein-Uhlenbeck case and thereby we contribute to the long problem of finding representation of $g_{b}^{0}$ via elementary functions. For this let us introduce some background of convolution algebra theory.

By $\mathcal{A}$ we denote the set of all real-valued locally integrable functions on $[0, \infty)$. The convolution of $f, g \in \mathcal{A}$ is given by (2.2). We recall after Lew (1972) that $\mathcal{A}$ is closed under point-wise addition, scalar multiplication and convolution. $\mathcal{A}$ is a commutative associative algebra over the real numbers, and we may define the convolution powers by

$$
f^{* 1}=f, \quad f^{*(n+1)}=f * f^{* n}, \quad n=1,2, \ldots .
$$


Let

$$
u_{t}(f)=\int_{0}^{t}|f(u)| d u, \quad t>0 .
$$

We denote by $\mathcal{U}$ the topology in $\mathcal{A}$ generated by the family of semi-norms $u_{n}(f), n=$ $1,2, \ldots$ The space $\mathcal{A}$ with this topology is complete, metrizable and locally convex. We have

$$
u_{t}(f * g) \leq u_{t}(f) u_{t}(g),
$$

and it is well known that the algebra $\mathcal{A}$ can be adjoined with an identity $\delta$ to obtain a topological algebra $\widehat{\mathcal{A}}=\mathcal{A} \oplus \delta$, called the unitization of $\mathcal{A}$ (see Naimark, 1959 p. 170). If $f, g_{1}, g_{2} \in \mathcal{A}, f \neq 0$ and $f * g_{1}=f * g_{2}$, it follows from Titchmarsh theorem that $g_{1}=g_{2}$ (see Titchmarsh, 1926).

For a given element $f \in \mathcal{A}$, one can define a quasi-inverse of $f$ which is an element $f^{\dagger} \in \mathcal{A}$ such that

$$
f+f^{\dagger}=f * f^{\dagger}
$$

It can be shown that each $f \in \mathcal{A}$ has a unique quasi-inverse, the mapping $f \mapsto f^{\dagger}$ defines a homeomorphism from $(\mathcal{A}, \mathcal{U})$ into itself (Lew, 1972, Theorem 1) and

$$
f^{\dagger}=-\sum_{n \geq 1} f^{* n}
$$

Hence, the sum on the RHS of the last equality always converges.

If $\delta$ denotes a neutral element, we have for any $h \in \mathcal{A}$ and quasi-inverse $h^{\dagger}$

$$
(\delta-h) *\left(\delta-h^{\dagger}\right)=\delta .
$$

Setting $\widehat{h}=\delta-h \in \widehat{\mathcal{A}}$ we see that $\widehat{h}$ is invertible in $\widehat{\mathcal{A}}$ and $\widehat{h}^{-1}=\delta-h^{\dagger}$. Finally, for any $f, g \in \mathcal{A}$ the Volterra integral equation of the second kind

$$
v=f+g * v
$$

has a unique solution given by

$$
v=f-f * g^{\dagger},
$$

which depends continuously on $f$ and $g$ in the topology $\mathcal{U}$ (see Lew, 1972, Theorem 2).

We are ready to present the announced formula for the density $g_{b}^{x}$.

Theorem 3.4. Assume that $x, b \in E$. For any $y \in E$ define

$$
\begin{aligned}
\Delta(x, y, b) & :=p(y, x)-p(y, x) *\left(-g_{x}^{b}\right)^{\dagger} \\
& =p(y, x)+p(y, x) * \sum_{n \geq 1}(-1)^{n}\left(g_{x}^{b}\right)^{* n} .
\end{aligned}
$$

Then

$$
g_{b}^{x}=\Delta(x, x, b) * \gamma_{x}+\Delta(x, x, b) * \sum_{n \geq 1} \Delta^{* n}(x, b, b) * \gamma_{x}^{*(n+1)} .
$$

Proof: By Corollary 3.3 we may write

$$
g_{b}^{x}=\widehat{p}^{(b)}(x, x) * \gamma_{x}-g_{b}^{x} * g_{x}^{b},
$$


and we look at the last equality as an integral equation with respect to $g_{b}^{x}$. Using (3.12) we obtain

$$
g_{b}^{x}=\widehat{p}^{(b)}(x, x) * \gamma_{x}-\widehat{p}^{(b)}(x, x) * \gamma_{x} *\left(-g_{x}^{b}\right)^{\dagger} .
$$

By the Hunt formula

$$
\widehat{p}^{(b)}(x, x)=p(x, x)-p(b, x) * g_{b}^{x},
$$

so inserting the last identity into (3.16) yields

$$
g_{b}^{x}=\left(p(x, x)-p(b, x) * g_{b}^{x}\right) * \gamma_{x}-\left(p(x, x)-p(b, x) * g_{b}^{x}\right) * \gamma_{x} *\left(-g_{x}^{b}\right)^{\dagger} .
$$

After reordering, we arrive at Volterra integral equation of the second kind with respect to $g_{b}^{x}$. Indeed, by the Hunt formula

$$
\begin{aligned}
g_{b}^{x} & =p(x, x) * \gamma_{x}-\left(p(x, x)-p(b, x) * g_{b}^{x}\right) *\left(-g_{x}^{b}\right)^{\dagger} * \gamma_{x}-g_{b}^{x} *\left(p(b, x) * \gamma_{x}\right) \\
& =p(x, x) * \gamma_{x}-p(x, x) *\left(-g_{x}^{b}\right)^{\dagger} * \gamma_{x}+g_{b}^{x} *\left(p(b, x) * \gamma_{x} *\left(-g_{x}^{b}\right)^{\dagger}-p(b, x) * \gamma_{x}\right) .
\end{aligned}
$$

It follows again from (3.12) that

$$
\begin{aligned}
g_{b}^{x} & =\left(p(x, x)-p(x, x) *\left(-g_{x}^{b}\right)^{\dagger}\right. \\
& \left.-\left(p(x, x)-p(x, x) *\left(-g_{x}^{b}\right)^{\dagger}\right) *\left(\gamma_{x} * p(b, x)-\gamma_{x} * p(b, x) *\left(-g_{x}^{b}\right)^{\dagger}\right)^{\dagger}\right) * \gamma_{x},
\end{aligned}
$$

so recalling the definition (3.13) of $\Delta$, we obtain from the last equality

$$
\begin{aligned}
g_{b}^{x} & =\left(\Delta(x, x, b)-\Delta(x, x, b) *\left(\gamma_{x} * \Delta(x, b, b)\right)^{\dagger}\right) * \gamma_{x} \\
& =\Delta(x, x, b) * \gamma_{x}-\Delta(x, x, b) * \gamma_{x} *\left(\gamma_{x} * \Delta(x, b, b)\right)^{\dagger} .
\end{aligned}
$$

The assertion follows from the definition of a quasi-inverse element.

Example 3.5. (Ornstein-Uhlenbeck process) Let $X$ be an Ornstein-Uhlenbeck process $(\mathrm{OU})$, that is a strong solution of $S D E$

$$
X_{t}=X_{0}+B_{t}-\alpha \int_{0}^{t} X_{u} d u,
$$

where $B$ is a standard Brownian motion. Alili, Patie and Pedersen (Alili et al., 2005) gather different expressions for the density function $g_{b}^{x}$ of the first hitting time of OU process. They provide the Laplace transform of $\sigma_{b}$ for $x<b\left(X_{0}=x\right)$ in terms of Hermite and parabolic cylinder functions, which is next inverted by means of the Cauchy residue theorem. The obtained denisty is a series expressed in terms of parabolic cylinder functions. Another representations given in Alili et al. (2005) is the cosine transform of the distribution of $\sigma_{b}$. As a result, the density $g_{b}^{x}$ is obtained out from the cosine transform. Their final method relies on observation that computing the density of $\sigma_{b}$ equals to characterizing the distribution of a quadratic functional of the 3-dimensional Bessel bridge. The killed version of the OU process is considered in Salminen et al. (2007), where the results rely on the use of Doob's transform and the form of density $g_{b}^{x}$ expressed as a limit with respect to scale function of the transition density of a killed process Salminen et al. (2007, (2)). In Lipton and Kaushansky (2018), author claimed that the problem of expressing $g_{b}^{x}(b \in \mathbb{R})$ in terms of elementary functions is still open. The newest result in this direction by Hamana (2020) concerns the first hitting time of radial OrnsteinUhlenbeck process (that is the euclidean norm of independent OU processes). He studies the case when the hitting site is closer to the origin than the starting point 
and obtains the probability density function by the Heaviside expansion theorem. Our approach, based on counting excursion technique, is new and totally different from all mentioned above.

As one can easily check (see Salminen et al., 2007, Sect. 5) all the ingredients of formula (3.14) for Ornstein-Uhlenbeck process are given. For simplicity we assume that $X_{0}=0$ and $\alpha>0$. Namely, we have for $x=0, b \in \mathbb{R}$ and $t>0$

$$
\begin{aligned}
p_{t}(0,0) & =\frac{1}{2} \frac{\sqrt{\alpha}}{\sqrt{2 \pi \sinh (\alpha t)}} e^{\frac{\alpha t}{2}}, \quad p_{t}(b, 0)=\frac{1}{2} \frac{\sqrt{\alpha}}{\sqrt{2 \pi \sinh (\alpha t)}} e^{\frac{\alpha t}{2}+\frac{\alpha b^{2}}{2}(1-\operatorname{coth}(\alpha t))}, \\
\gamma_{0}(d t) & =\frac{\alpha^{3 / 2} e^{\frac{\alpha t}{2}}}{\sqrt{2 \pi}(\sinh (\alpha t))^{3 / 2}} d t, \quad g_{0}^{b}(t)=b \gamma_{0}(t) e^{-b \frac{\alpha e^{-\alpha t}}{2 \sinh (\alpha t)}},
\end{aligned}
$$

and so

$$
g_{b}^{0}=\Delta(0,0, b) * \gamma_{0}+\Delta(0,0, b) * \sum_{n \geq 1} \Delta^{* n}(0, b, b) * \gamma_{0}^{*(n+1)}
$$

where

$$
\begin{aligned}
& \Delta(0,0, b)=p(0,0)+p(0,0) * \sum_{n \geq 1}(-1)^{n}\left(g_{0}^{b}\right)^{* n}, \\
& \Delta(0, b, b)=p(b, 0)+p(b, 0) * \sum_{n \geq 1}(-1)^{n}\left(g_{0}^{b}\right)^{* n} .
\end{aligned}
$$

The above asymmetries motivate a question about the similar effects within the class of additive functionals of $X$. The question is how the symmetry of an additive functional $F$ on a canonical space between expectations of minimal processes affects the expectations of the same additive functional but on the path-space with respect to the excursion measure. The next result shows the dependencies between expectations of such a functional taken with respect to $\widehat{\mathbb{P}}_{x}$ and $\widehat{\mathbb{P}}_{b}$ respectively. We consider $F$ defined on a path-space of continuous functions.

Definition 3.6. We say that there is an universal symmetry for $x, b \in E$ and $F$ if

$$
\mathbb{E}_{x} F_{t}\left(\widehat{X}^{(b)}\right)=\mathbb{E}_{b} F_{t}\left(\widehat{X}^{(x)}\right), \quad t>0,
$$

where the expectations above are assumed to be finite.

As usual $\widehat{X}^{(b)}$ denotes the process killed at first hitting time on $b$. We say that $F$ is universally integrable if

$$
\mathbb{E}_{x} F_{t}\left(\widehat{X}^{(y)}\right)<\infty, \quad \widehat{\mathbb{P}}_{x} F_{t \wedge R}(X)<\infty,
$$

for any $x, y \in E$ and $t>0$. We say that $F$ is of finite potential if for every $\lambda>0$

$$
\mathbb{E}_{x} \int_{0}^{\infty} e^{-\lambda t} d F_{t}(X)<\infty, \quad x, y \in E .
$$

For $t>0$ we define two measures

$$
\mu_{x, b}(0, t):=\mathbb{E}_{x} F_{t}\left(\widehat{X}^{(b)}\right), \quad \widehat{\mu}_{x}(0, t):=\widehat{\mathbb{P}}_{x} F_{t \wedge R}(X),
$$

where in the second definition $X$ denotes a coordinate process on the path-space $U$. Since $F_{0}=0$, it is enough to define above measures on open intervals $(0, t), t>0$. By equality $\mu_{x, b}=\mu_{b, x}\left(\widehat{\mu}_{x}=\widehat{\mu}_{b}\right)$ we mean $\mu_{x, b}(0, t)=\mu_{b, x}(0, t)\left(\widehat{\mu}_{x}(0, t)=\widehat{\mu}_{b}(0, t)\right)$ for every $t>0$. 
Theorem 3.7. Assume that $\left(F_{t}\right)_{t>0}$ is an additive and universally integrable functional of $X$, and that there is an universal symmetry for $x, b$ and $F$. Then

(1) $\left(1+g_{b}^{x}\right) * p(b, b) * \widehat{\mu}_{b}=\left(1+g_{x}^{b}\right) * p(x, x) * \widehat{\mu}_{x}$.

(2) $\widehat{\mu}_{x}=\widehat{\mu}_{b} \quad \Leftrightarrow p(x, x)=p(b, b)$.

Proof: Step 1. Assume that (3.17) holds for fixed $x, b \in E$ and every $t>0$. Let $A_{t}:=F_{t}(X)$ and $\widehat{A}_{t}^{(b)}:=F_{t}\left(\widehat{X}^{(b)}\right)$. For $\lambda>0$ consider $\mathbb{E}_{x} \int_{0}^{t \wedge \sigma_{b}} e^{-\lambda u} d A_{u}$. Repeating the idea of proof of Theorem 3.1 we obtain $I_{t}-I I_{t}=I I I_{t}$, where

$$
\begin{aligned}
I_{t} & :=\mathbb{E}_{x} \sum_{\tau_{s-}^{x}<t \wedge \sigma_{b}} e^{-\lambda \tau_{s-}^{x}}\left(\int_{0}^{\sigma_{x}} e^{-\lambda u} d A_{u}\right) \circ \theta_{\tau_{s-}^{x}}, \\
I I_{t} & :=\mathbb{E}_{x}\left(e^{-\lambda\left(t \wedge \sigma_{b}\right)} \mathbb{E}_{X_{t \wedge \sigma_{b}}} \int_{0}^{\sigma_{x}} e^{-\lambda u} d A_{u}\right), \quad I I I_{t}:=\mathbb{E}_{x} \int_{0}^{t} e^{-\lambda u} d \widehat{A}_{u}^{(b)} .
\end{aligned}
$$

Using the master formula of excursions we obtain

$$
I_{t}=\mathbb{E}_{x} \int_{0}^{t} e^{-\lambda s} d L_{\sigma_{b} \wedge s}^{x} \int_{U} \bar{F}(u) \widehat{\mathbb{P}}_{x}(d u)
$$

where $\bar{F}$ is defined on the path-space $U$ by $\bar{F}(u)=\int_{0}^{R(u)} e^{-\lambda s} d F_{s}(u)$. Putting the things together we conclude that

$$
\begin{aligned}
\mathbb{E}_{x} \int_{0}^{t} e^{-\lambda s} d L_{\sigma_{b} \wedge s}^{x} \int_{U} \bar{F}(u) \widehat{\mathbb{P}}_{x}(d u) & =\mathbb{E}_{x}\left(e^{-\lambda\left(t \wedge \sigma_{b}\right)} \mathbb{E}_{X_{t \wedge \sigma_{b}}} \int_{0}^{\sigma_{x}} e^{-\lambda u} d A_{u}\right) \\
& +\mathbb{E}_{x} \int_{0}^{t} e^{-\lambda u} d \widehat{A}_{u}^{(b)} .
\end{aligned}
$$

Recall that $\mu_{x, b}(0, s):=\mathbb{E}_{x} \widehat{A}_{s}^{(b)}$ and $\widehat{\mu}_{x}(0, s):=\widehat{\mathbb{P}}_{x} F_{s \wedge R}(X)$. Moreover,

$$
\mathbb{E}_{x}\left(e^{-\lambda t} 1_{\left\{t<\sigma_{b}\right\}} \mathbb{E}_{X_{t}} \int_{0}^{\sigma_{x}} e^{-\lambda u} d A_{u}\right) \leq e^{-\lambda t} \mathbb{E}_{x} \int_{t}^{\infty} e^{-\lambda u} d A_{u},
$$

so by the finiteness of the potential of $A$, the last tends to 0 with $t \rightarrow \infty$. By (3.18) and arbitrary choice of $\lambda$

$$
\int_{0}^{t} \widehat{p}_{t-s}^{(b)}(x, x) \widehat{\mu}_{x}(d s)=\int_{0}^{t} g_{b}^{x}(t-s) \mu_{b, x}(d s)+\mu_{x, b}(0, t),
$$

where we used the identity $\int_{0}^{t} \widehat{p}_{s}^{(b)}(x, x) d s=\mathbb{E}_{x} L_{\sigma_{b} \wedge t}^{x}$. Let $\bar{\mu}_{x, b}(t):=\mu_{x, b}(0, t)$. Rewriting (3.19) in terms of convolution algebra yields

$$
\widehat{p}^{(b)}(x, x) * \widehat{\mu}_{x}=\bar{\mu}_{x, b}+g_{b}^{x} * \mu_{b, x} .
$$

Since $\bar{\mu}_{x, b}=1 * \mu_{x, b}$ and $\mu_{x, b}=\mu_{b, x}$ (by universal symmetry), we may rewrite the last equality as

$$
\widehat{p}^{(b)}(x, x) * \widehat{\mu}_{x}=\left(1+g_{b}^{x}\right) * \mu_{b, x},
$$

and analogously

$$
\widehat{p}^{(x)}(b, b) * \widehat{\mu}_{b}=\left(1+g_{x}^{b}\right) * \mu_{x, b} .
$$

Using the Hunt formula and multiplying both sides by $p(b, b)$ (in a sense of convolution) yield

$$
(p(b, b) * p(x, x)-p(b, x) * p(b, x)) * \widehat{\mu}_{x}=p(b, b) *\left(1+g_{b}^{x}\right) * \mu_{b, x},
$$


and by symmetry

$$
(p(b, b) * p(x, x)-p(b, x) * p(b, x)) * \widehat{\mu}_{b}=p(x, x) *\left(1+g_{x}^{b}\right) * \mu_{b, x} .
$$

Thus

$$
p(b, b) *\left(1+g_{b}^{x}\right) * \widehat{\mu}_{b}=p(x, x) *\left(1+g_{x}^{b}\right) * \widehat{\mu}_{x} .
$$

Step 2. $(\Rightarrow)$ If $\widehat{\mu}_{x}=\widehat{\mu}_{b}$, we have from point 1 .

$$
p(b, b) *\left(1+g_{b}^{x}\right)=p(x, x) *\left(1+g_{x}^{b}\right),
$$

from which it follows that

$$
p(b, b) * 1+p(x, b)=p(x, x) * 1+p(b, x) .
$$

Symmetry of $p$ yields that $p(x, x)=p(b, b)$.

$(\Leftarrow)$ Observe that for given $x, b \in E$ equality $p(x, x)=p(b, b)$ is equivalent with equality $g_{b}^{x}=g_{x}^{b}$. Indeed, this follows from Corollary 3.2. If $p(x, x)=p(b, b)$, then by equality (3.7) we have $\left(g_{x}^{b}-g_{b}^{x}\right) * p(x, x)=0$, so $g_{b}^{x}=g_{x}^{b}$. If in turn $g_{b}^{x}=g_{x}^{b}$, then again by (3.7) it follows that $\widehat{p}^{(b)}(x, x)-\widehat{p}^{(x)}(b, b)=0$, so by the Hunt formula we immediately get $p(x, x)-p(b, b)=0$. Hence, by point 1 . we easily find that $p(x, x)=p(b, b)$ implies $\widehat{\mu}_{x}=\widehat{\mu}_{b}$.

Remark 3.8. If we define an operator $\mathcal{K}_{x, b}: \mathcal{A} \rightarrow \mathcal{A}$ by

$$
\mathcal{K}_{x, b} f=f *\left(1+g_{b}^{x}\right) * p(b, b), \quad f \in \mathcal{A},
$$

then by point 1 . of Theorem 3.7 and Fubini's theorem, we conclude that an universal symmetry for $x, b$ and $F$ implies that for each $f \in \mathcal{A}$

$$
\widehat{\mathbb{P}}_{x} \int_{0}^{t} \mathcal{K}_{x, b} f(t-u) d F_{u \wedge R}(X)=\widehat{\mathbb{P}}_{b} \int_{0}^{t} \mathcal{K}_{b, x} f(t-u) d F_{u \wedge R}(X) .
$$

Example 3.9. The elementary example of situation $p(x, x)-p(b, b)=0$ is the case of $p$ being the transition density of a Brownian motion. Indeed, the known identity $\mathbb{E}_{x} e^{-\lambda \sigma_{b}}=e^{-\lambda|x-b|}$ confirms the symmetry and equality $g_{b}^{x}=g_{x}^{b}$.

Next, we define a notion of strong universal symmetry for a "classical" additive functional and show that it is not satisfied unless $p(x, x)-p(b, b)=0$.

Definition 3.10. For a positive Borel function $f$ on $E$ we define on a functional $F^{f}$ by

$$
F_{t}^{f}(X):=\int_{0}^{t} f\left(X_{u}\right) d u
$$

We say that a strong universal symmetry holds for $x, b \in E$ if there is an universal symmetry for $x, b$ and $F^{f}$ for each positive Borel $f$.

Corollary 3.11. There is no strong universal symmetry for $x, b \in E$ unless $p(x, x)-p(b, b)=0$.

Proof: If strong universal symmetry holds for $x, b \in E$ then $\mathbb{E}_{x} F_{t}^{f}\left(\widehat{X}^{(b)}\right)=$ $\mathbb{E}_{b} F_{t}^{f}\left(\widehat{X}^{(x)}\right)$ for each $t>0$ and each positive bounded Borel $f$ on $E$. Thus $\widehat{p}_{t}^{(b)}(x, z)=\widehat{p}_{t}^{(x)}(b, z)$ each $z \in E$ and integrating out with respect to $m(d z)$ shows that $\mathbb{P}_{x}\left(\sigma_{b}>t\right)=\mathbb{P}_{b}\left(\sigma_{x}>t\right)$. Hence, $g_{b}^{x}=g_{x}^{b}$ and we conclude by Theorem 3.7 that $p(x, x)=p(b, b)$. 
If we assume a little bit more about the function $t \mapsto g_{b}^{x}$, we obtain a symmetric formula joining $\widehat{\mu}_{x}, \widehat{\mu}_{b}$ and $g_{b}^{x}, g_{x}^{b}$.

Theorem 3.12. Under assumptions of Theorem 3.7 suppose that $g_{b}^{x}, g_{x}^{b} \in \mathcal{C}^{1}(0, \infty)$. Then

$$
\left(\frac{\partial g_{x}^{b}}{\partial t}+\frac{\partial g_{x}^{b}}{\partial t} * \frac{\partial g_{b}^{x}}{\partial t}\right) * \widehat{\mu}_{b}=\left(\frac{\partial g_{b}^{x}}{\partial t}+\frac{\partial g_{x}^{b}}{\partial t} * \frac{\partial g_{b}^{x}}{\partial t}\right) * \widehat{\mu}_{x}
$$

Moreover

$$
g_{x}^{b} * \widehat{\mu}_{b}=\left(1+g_{x}^{b}\right) *\left(-\frac{\partial g_{b}^{x}}{\partial t}\right)^{\dagger} * \widehat{\mu}_{x}
$$

Proof: It is well known that for a kernel $k$ on $[0, \infty)$ such that $k(0)=1$, the Volterra equation of the first kind $y * k=f$ (with respect to $y$ ) for $k, f \in \mathcal{C}^{1}(0, \infty)$ may be reduced to the Volterra equation of the second kind $y+\frac{\partial k}{\partial t} * y=\frac{\partial f}{\partial t}$ (see Gripenberg et al., 1990 or Friedman and Shinbrot, 1967). Hence, $y=\frac{\partial f}{\partial t}-\frac{\partial f}{\partial t} *\left(-\frac{\partial k}{\partial t}\right)^{\dagger}$. Since $g_{b}^{x}, g_{x}^{b} \in \mathcal{C}^{1}(0, \infty)$, we may consider the integral equation

$$
1 * p(b, b) * \widehat{\mu}_{b} *\left(1+g_{b}^{x}\right)=1 * p(x, x) * \widehat{\mu}_{x} *\left(1+g_{x}^{b}\right),
$$

with $1+g_{b}^{x}$ taken as a kernel $k$, the RHS of (3.24) taken as $f$ and $y:=1 * p(b, b) * \widehat{\mu}_{b}$. Indeed, the regularity of points $x, b$ implies that $k(0)=1$. The rest of assumptions follows easily. We solve the equation and obtain

$$
1 * p(b, b) * \widehat{\mu}_{b}=p(x, x) * \widehat{\mu}_{x} *\left(1+g_{x}^{b}\right)-p(x, x) * \widehat{\mu}_{x} *\left(1+g_{x}^{b}\right) *\left(-\frac{\partial g_{b}^{x}}{\partial t}\right)^{\dagger} .
$$

From point 1. of Theorem 3.7 and the last identity

$$
\begin{aligned}
p(x, b) * \widehat{\mu}_{b} & +p(x, x) * \widehat{\mu}_{x} *\left(1+g_{x}^{b}\right)-p(x, x) * \widehat{\mu}_{x} *\left(1+g_{x}^{b}\right) *\left(-\frac{\partial g_{b}^{x}}{\partial t}\right)^{\dagger} \\
& =p(x, x) * \widehat{\mu}_{x} *\left(1+g_{x}^{b}\right) .
\end{aligned}
$$

As a result

$$
p(x, b) * \widehat{\mu}_{b}=p(x, x) * \widehat{\mu}_{x} *\left(1+g_{x}^{b}\right) *\left(-\frac{\partial g_{b}^{x}}{\partial t}\right)^{\dagger},
$$

and (3.23) follows from the last equality and identity $p(x, b)=p(x, x) * g_{x}^{b}$. Next, we rewrite (3.23) as

$$
1 * \frac{\partial g_{x}^{b}}{\partial t} * \widehat{\mu}_{b}=1 *\left(-\frac{\partial g_{b}^{x}}{\partial t}\right)^{\dagger} * \widehat{\mu}_{x}+1 * \frac{\partial g_{x}^{b}}{\partial t} *\left(-\frac{\partial g_{b}^{x}}{\partial t}\right)^{\dagger} * \widehat{\mu}_{x}
$$

Multiplying both sides of the last equality in a sense of convolution by $-\frac{\partial g_{b}^{x}}{\partial t}$ yields

$$
-\frac{\partial g_{b}^{x}}{\partial t} * \frac{\partial g_{x}^{b}}{\partial t} * \widehat{\mu}_{b}=\frac{\partial g_{x}^{b}}{\partial t} * \widehat{\mu}_{b}+\left(\widehat{\mu}_{x}+\frac{\partial g_{x}^{b}}{\partial t} * \widehat{\mu}_{x}\right) *\left(-\frac{\partial g_{b}^{x}}{\partial t}\right),
$$

and equality (3.23) follows from the last equality and some simple algebra. 


\section{Stopping at exit time}

In the previous section, for a given $b \in E$, we counted excursions before $t \wedge \sigma_{b}$ and obtained a number of identities for a diffusion stopped at the first hitting time of a single point. In what follows we will run analogous study for a diffusion stopped at the first exit time from a given interval. We will count the excursions of $X$ from $x$ and stop the process at $\sigma_{I}:=\sigma_{a} \wedge \sigma_{b}$, where $x \in I=(a, b)$. We introduce an auxiliary notation. For $a<x<b$ we denote

$$
g_{a \mid b}^{x}(t) d t:=\mathbb{P}_{x}\left(\sigma_{a} \in d t, \sigma_{a}<\sigma_{b}\right), \quad g_{b \mid a}^{x}(t) d t:=\mathbb{P}_{x}\left(\sigma_{b} \in d t, \sigma_{b}<\sigma_{a}\right) .
$$

By $\widehat{p}_{t}^{(I)}$ we denote a transition density with respect to speed measure of a diffusion killed at first exit from $I$, namely

$$
\widehat{p}_{t}^{(I)}(x, z) m(d z)=\mathbb{P}_{x}\left(X_{t} \in d z, t<\sigma_{I}\right) .
$$

For objects used before we use the previous notation.

Theorem 4.1. Assume that $x \in I=(a, b)$. For any $z \in I$

$$
\widehat{p}^{(x)}(a, z) * g_{a \mid b}^{x}+\widehat{p}^{(x)}(b, z) * g_{b \mid a}^{x}=g_{x}^{z} * \widehat{p}^{(I)}(x, x)-\widehat{p}^{(I)}(x, z) .
$$

Proof: Let $t>0$. The proof relies on counting excursions, this time before $\sigma_{I} \wedge$ $t$. We will skip the repetitions. Let $\lambda>0$ and $f$ be a positive and bounded Borel function on $E$. As always we define a function on a path space $F_{\lambda}(u)=$ $\int_{0}^{R(u)} e^{-\lambda s} f(u(s)) d s$. What we get from counting excursions approach is equality $I I_{t}=I_{t}-I I I_{t}$, where

$$
\begin{aligned}
& I_{t}:=\mathbb{E}_{x} \int_{0}^{t} e^{-\lambda s} d L_{s \wedge \sigma_{I}}^{x} \int_{U} F_{\lambda}(u) \widehat{\mathbb{P}}_{x}(d u), \\
& I I_{t}:=\mathbb{E}_{x}\left(e^{-\lambda t \wedge \sigma_{I}} \mathbb{E}_{X_{t \wedge \sigma_{I}}} \int_{0}^{\sigma_{x}} e^{-\lambda s} f\left(X_{s}\right) d s\right) \\
& =\mathbb{E}_{x}\left(1_{\left\{\sigma_{a}<\sigma_{b}\right\}} e^{-\lambda t \wedge \sigma_{a}} \mathbb{E}_{a} \int_{0}^{\sigma_{x}} e^{-\lambda s} f\left(X_{s}\right) d s+1_{\left\{\sigma_{a}>\sigma_{b}\right\}} e^{-\lambda t \wedge \sigma_{b}} \mathbb{E}_{b} \int_{0}^{\sigma_{x}} e^{-\lambda s} f\left(X_{s}\right) d s\right), \\
& I I I_{t}:=\mathbb{E}_{x} \int_{0}^{\sigma_{I} \wedge t} e^{-\lambda s} f\left(X_{s}\right) d s=\int_{E} \int_{0}^{t} e^{-\lambda s} f(z) \widehat{p}_{s}^{(I)}(x, z) d s m(d z) .
\end{aligned}
$$

Introducing $\bar{\mu}_{x, I}(t):=\mathbb{E}_{x} L_{t \wedge \sigma_{I}}^{x}$ and using the same arguments like in proof of Theorem 3.1 we conclude that

$$
1 * \widehat{p}^{(x)}(a, z) * g_{a \mid b}^{x}+1 * \widehat{p}^{(x)}(b, z) * g_{b \mid a}^{x}=g_{x}^{z} * \bar{\mu}_{x, I}-1 * \widehat{p}^{(I)}(x, z) .
$$

By occupation time formula

$$
\mathbb{E}_{x} \int_{0}^{t} f\left(\widehat{X}_{s}^{(I)}\right) d s=\mathbb{E}_{x} \int_{E} f(z) L_{t \wedge \sigma_{I}}^{z} m(d z),
$$

so $\bar{\mu}_{x, I}=1 * \widehat{p}^{(I)}(x, x)$. As a result, we rewrite (4.2) as follows

$$
1 * \widehat{p}^{(x)}(a, z) * g_{a \mid b}^{x}+1 * \widehat{p}^{(x)}(b, z) * g_{b \mid a}^{x}=g_{x}^{z} * 1 * \widehat{p}^{(I)}(x, x)-1 * \widehat{p}^{(I)}(x, z),
$$

which concludes the proof.

Taking limits $z \uparrow b$ and $z \downarrow a$ in formula (4.1) yield a counterpart to Corollary 3.2. 
Corollary 4.2. For $a<x<b$

$$
\begin{aligned}
& \widehat{p}^{(x)}(a, b) * g_{a \mid b}^{x}+\widehat{p}^{(x)}(b, b) * g_{b \mid a}^{x}=g_{x}^{b} * \widehat{p}^{(I)}(x, x), \\
& \widehat{p}^{(x)}(a, a) * g_{a \mid b}^{x}+\widehat{p}^{(x)}(b, a) * g_{b \mid a}^{x}=g_{x}^{a} * \widehat{p}^{(I)}(x, x) .
\end{aligned}
$$

Corollary 4.3. Let $\gamma_{x}(d t)=\widehat{\mathbb{P}}_{x}(R \in d t)$. Then

$$
\mathbb{P}_{x}\left(\sigma_{I} \in d t\right)+\mathbb{P}_{x}\left(\sigma_{I}+\sigma_{x} \circ \theta_{\sigma_{I}} \in d t\right)=\left(\widehat{p}^{(I)}(x, x) * \gamma_{x}\right)(t) d t .
$$

Proof: We integrate both sides of (4.1) with respect to $m(d z)$ and recall that $g_{x}^{z}(t) m(d z)=\widehat{\mathbb{P}}_{x}\left(\widehat{X}_{t} \in d z\right)(\widehat{X}$ is a coordinate process on path-space killed at $R$ ). Recall that $m$ does not charge any single point. By a little effort one can verify that $\sigma_{I}$ has a density denoted (under $\mathbb{P}_{x}$ ) by $g_{I}^{x}$. We obtain

$$
g_{I}^{x}+g_{x}^{a} * g_{a \mid b}^{x}+g_{x}^{b} * g_{b \mid a}^{x}=\widehat{p}^{(I)}(x, x) * \gamma_{x} .
$$

Next, by strong Markov property

$$
\left(\int_{0}^{t} g_{x}^{a}(t-u) \mathbb{P}_{x}\left(\sigma_{a} \in d u, \sigma_{a}<\sigma_{b}\right)\right) d t=\mathbb{P}_{x}\left(\sigma_{a}+\sigma_{x} \circ \theta_{\sigma_{a}} \in d t, \sigma_{a}<\sigma_{b}\right),
$$

so we may rewrite the LHS of (4.3) as

$$
\begin{aligned}
g_{I}^{x}(t) d t+\mathbb{P}_{x}\left(\sigma_{a}+\sigma_{x} \circ \theta_{\sigma_{a}} \in d t, \sigma_{a}<\sigma_{b}\right) & +\mathbb{P}_{x}\left(\sigma_{b}+\sigma_{x} \circ \theta_{\sigma_{b}} \in d t, \sigma_{b}<\sigma_{a}\right) \\
& =g_{I}^{x}(t) d t+\mathbb{P}_{x}\left(\sigma_{I}+\sigma_{x} \circ \theta_{\sigma_{I}} \in d t\right) .
\end{aligned}
$$

The assertion follows.

The next identity links the joint distribution of $X_{t}$ and exit time from $I$, with the entrance law of excursions.

Theorem 4.4. Let $x \in(a, b)$. For any $t>0$ we have on $I$

$$
\mathbb{P}_{x}\left(X_{t} \in d z, \sigma_{I}+\sigma_{x} \circ \theta_{\sigma_{I}}>t\right)=\int_{0}^{t} \widehat{p}_{t-u}^{(I)}(x, x) \widehat{\mathbb{P}}_{x}\left(\widehat{X}_{u} \in d z\right) d u .
$$

Proof: Formula (4.1) yields

$$
\begin{aligned}
& \int_{0}^{t} \mathbb{P}_{a}(\left.X_{t-u} \in d z, t-u<\sigma_{x}\right) \mathbb{P}_{x}\left(\sigma_{a} \in d u, \sigma_{a}<\sigma_{b}\right) \\
& \quad+\int_{0}^{t} \mathbb{P}_{b}\left(X_{t-u} \in d z, t-u<\sigma_{x}\right) \mathbb{P}_{x}\left(\sigma_{b} \in d u, \sigma_{b}<\sigma_{a}\right) \\
& \quad+\mathbb{P}_{x}\left(X_{t} \in d z, \sigma_{I}>t\right)=\int_{0}^{t} \widehat{p}_{t-u}^{(I)}(x, x) g_{x}^{z}(u) m(d z) d u .
\end{aligned}
$$

Using strong Markov property and the form of entrance law, we rewrite the last equality

$$
\begin{aligned}
& \int_{0}^{t} \mathbb{P}_{x}(\left.X_{t-u+\sigma_{a}} \in d z, \sigma_{a} \in d u, t-u<\sigma_{x} \circ \theta_{\sigma_{a}}, \sigma_{a}<\sigma_{b}\right) \\
& \quad+\int_{0}^{t} \mathbb{P}_{x}\left(X_{t-u+\sigma_{b}} \in d z, \sigma_{b} \in d u, t-u<\sigma_{x} \circ \theta_{\sigma_{b}}, \sigma_{b}<\sigma_{a}\right) \\
& \quad+\mathbb{P}_{x}\left(X_{t} \in d z, \sigma_{I}>t\right)=\int_{0}^{t} \widehat{p}_{t-u}^{(I)}(x, x) \widehat{\mathbb{P}}_{x}\left(\widehat{X}_{u} \in d z\right) d u
\end{aligned}
$$


To conclude the assertion it is enough to observe that

$$
\begin{aligned}
\mathbb{P}_{x}\left(X_{t} \in d z, \sigma_{a}<t<\sigma_{a}\right. & \left.+\sigma_{x} \circ \theta_{\sigma_{a}}, \sigma_{a}<\sigma_{b}\right) \\
& +\mathbb{P}_{x}\left(X_{t} \in d z, \sigma_{b}<t<\sigma_{b}+\sigma_{x} \circ \theta_{\sigma_{b}}, \sigma_{b}<\sigma_{a}\right) \\
& =\mathbb{P}_{x}\left(X_{t} \in d z, \sigma_{I}<t<\sigma_{I}+\sigma_{x} \circ \theta_{\sigma_{I}}\right) .
\end{aligned}
$$

Notice that we have already found the description of the process built on $X$, which starts from $x$, and after exiting the interval $I$, is killed at the first hitting of $x$. In other words, the new process is killed at the stopping time defined by $d_{\sigma_{I}}^{x}=\inf \left\{t>\sigma_{I}: X_{t}=x\right\}$. Such a construction is popular in mathematical finance for modeling a continuous time barrier, and we name it here double-knockin construction after that. Till the exit from $I$, the process behaves like $X$ and after then becomes a new process killed at the first hitting of $x$. Since we assume the infinite life-time of $X$, we may conclude the same property for one barrier and obtain so called knock-in construction. We start defining a new process

$$
\widehat{X}_{t}^{I \rightarrow x}:=X_{t} 1_{\left\{t<d_{\sigma_{I}}^{x}\right\}}+\partial 1_{\left\{t \geq d_{\sigma_{I}}^{x}\right\}}, \quad t \geq 0,
$$

where $\partial$ is the cemetery state. Clearly $\mathbb{P}_{x}\left(\widehat{X}_{t}^{I \rightarrow x} \in d z\right)=\mathbb{P}_{x}\left(X_{t} \in d z, t<d_{\sigma_{I}}^{x}\right)$.

Corollary 4.5. The process $\left(\widehat{X}^{I \rightarrow x}\right)$ is under $\mathbb{P}_{x}$ a strong Markov process with the transition density (with respect to speed measure $m$ ) given by

$$
\widehat{p}_{t}^{I \rightarrow x}(x, z)=\left(g_{x}^{z} * \widehat{p}^{(I)}(x, x)\right)(t), \quad t \geq 0, z \in E .
$$

Proof: We find a candidate for the transition density

$$
\mathbb{P}_{x}\left(X_{t} \in d z, d_{\sigma_{I}}^{x}>t\right)=\left(\int_{0}^{t} \widehat{p}_{t-u}^{(I)}(x, x) g_{x}^{z}(u) d u\right) m(d z) .
$$

Take $s, t>0$. Since $d_{\sigma_{I}}^{x}$ is a terminal stopping time, we find using Markov property that

$$
\begin{aligned}
\mathbb{P}_{x}\left(\widehat{X}_{s}^{I \rightarrow x} \in d v, \widehat{X}_{t+s}^{I \rightarrow x} \in d z\right) & =\mathbb{P}_{x}\left(X_{s} \in d v, s<d_{\sigma_{I}}^{x}, X_{t+s} \in d z, t+s<d_{\sigma_{I}}^{x}\right) \\
& =\mathbb{E}_{x}\left(1_{\left\{X_{s} \in d v, s<d_{\sigma_{I}}^{x}\right\}} \mathbb{P}_{X_{s}}\left(X_{t} \in d z, t<d_{\sigma_{I}}^{x}\right)\right) .
\end{aligned}
$$

Using twice (4.6), we conclude that the last expression is equal to

$$
\begin{aligned}
\mathbb{E}_{x}\left(1_{\left\{X_{s} \in d v, s<d_{\sigma_{I}}^{x}\right\}}\right. & \left.\left(\int_{0}^{t} \widehat{p}_{t-u}^{(I)}\left(X_{s}, X_{s}\right) g_{X_{s}}^{z}(u) d u\right) m(d z)\right) \\
& =\left(\int_{0}^{t} \widehat{p}_{t-u}^{(I)}(v, v) g_{v}^{z}(u) d u\right) m(d z)\left(\int_{0}^{s} \widehat{p}_{s-u}^{(I)}(x, x) g_{x}^{v}(u) d u\right) m(d v) \\
& =\widehat{p}_{s}^{I \rightarrow x}(x, v) \widehat{p}_{t}^{I \rightarrow x}(v, z) m(d z) m(d v) .
\end{aligned}
$$

Due to the infinite life-time, we may consider $b$ at the right-end of the state space (and $a$ at the left-end) and by limiting $\sigma_{b} \rightarrow \infty$ (and $\sigma_{a} \rightarrow \infty$ ) we obtain two strong Markov processes $\widehat{X}^{a \rightarrow x}, \widehat{X}^{b \rightarrow x}$ (of knock-in type) killed at the first hitting of $x$, just after hitting the single barrier. The formulas below generalize the Hunt formula. 
Corollary 4.6. Let $a<x<b$. The transition densities of $\widehat{X}^{a \rightarrow x}, \widehat{X}^{b \rightarrow x}$ are given respectively by

$$
\begin{array}{ll}
\widehat{p}^{a \rightarrow x}(x, z)=p(x, z)-p(a, x) * g_{a}^{x} * g_{x}^{z}, & z \in E \\
\widehat{p}^{b \rightarrow x}(x, z)=p(x, z)-p(b, x) * g_{b}^{x} * g_{x}^{z}, & z \in E .
\end{array}
$$

Proof: Follows from (4.5), taking $b$ to the limit (and $a$ respectively), and then using Hunt formula.

\section{Excursion local time}

One of the advantages of the choice of a diffusive setup is that in line with Trotter's approach, based on deterministic occupation time of a well measurable set, we define the excursion local time. For any Borel non-negative $f$ on $E$ we define a function $(t, y) \mapsto \widehat{L}_{t}(y)$ by

$$
\int_{0}^{t \wedge R} f(u(s)) d s=\int_{E} f(y) \widehat{L}_{t}(y) m(d y), \quad t \geq 0, u \in U .
$$

In what follows, we assume that $\widehat{L}_{t}(y)$ is a right-continuous function of $y$ and continuous of $t$. Moreover, it is an additive functional, non-decreasing with respect to $t$ (see the discussion in Blumenthal, 1992, Chap. VI, Sect. 3). This is exactly what one expects the path-wise local time inherits from the probabilistic one. By identifying excursion space with a subspace of the canonical space, we may conclude the probabilistic definition of the local time

$$
\int_{0}^{t} f\left(\widehat{X}_{s}\right) d s=\int_{E} f(y) \widehat{L}_{t}(y) m(d y), \quad t \geq 0,
$$

where $m$ denotes the speed measure of $X$ (see also Salminen et al., 2007, p.5).

To sum up, we assume that on the excursion space we are given a local time defined as an additive functional on the canonical space. We apply counting excursions to obtain a relation between excursion local time and the measure induced by Laplace transforms of the local time of $X$. To establish the main result of this section we will need two lemmas. Notice that $\widehat{\mathbb{E}}_{x}$ denotes the expectation taken with respect to the excursion measure $\widehat{\mathbb{P}}_{x}$.

Lemma 5.1. Let $t>0$. Then

$$
\int_{0}^{t} \widehat{\mathbb{P}}_{x}(R>s) d s=\widehat{\mathbb{E}}_{x}(R \wedge t)<\infty .
$$

Proof: It is shown in Knight (1981) that $\widehat{\mathbb{P}}_{x}(R>s)$ is the Laplace transform of a measure $\mu$ on $[0, \infty)$ and absolutely continuous with a smooth and monotone decreasing density. Thus

$$
\int_{0}^{t} \widehat{\mathbb{P}}_{x}(R>s) d s=t \widehat{\mathbb{P}}_{x}\left(R>t_{0}\right)
$$

for some $t_{0} \in(0, t)$.

Lemma 5.2. For fixed $x, y \in E$ and $\gamma>0$

$$
\widehat{\mathbb{E}}_{x} e^{-\gamma \widehat{L}_{t}(y)}<\infty .
$$


Proof: Not losing generality assume that $t=1$. Since we defined $\widehat{L}_{1}(y)$ by the occupation time formula, we may easily conclude that

$$
\widehat{L}_{1}(y)=\lim _{\epsilon \downarrow 0} \frac{1}{m((y-\epsilon, y+\epsilon))} \int_{0}^{1} 1_{(y-\epsilon, y+\epsilon)}\left(\widehat{X}_{s}\right) d s .
$$

Thus, by Fatou lemma

$$
\widehat{\mathbb{E}}_{x} e^{-\gamma \widehat{L}_{1}(y)} \leq \lim _{\epsilon \downarrow 0} \widehat{\mathbb{E}}_{x} \exp \left\{-\frac{\gamma}{m((y-\epsilon, y+\epsilon))} \int_{0}^{1} 1_{(y-\epsilon, y+\epsilon)}\left(\widehat{X}_{s}\right) d s\right\} .
$$

By Jensen inequality and Fubini's theorem we conclude that the last limit is bounded by

$$
\lim _{\epsilon \downarrow 0} \int_{0}^{1} \widehat{\mathbb{E}}_{x} \exp \left\{-\frac{\gamma}{m((y-\epsilon, y+\epsilon))} 1_{(y-\epsilon, y+\epsilon)}\left(\widehat{X}_{s}\right)\right\} d s=: J .
$$

Using again the form of entrance law for $\widehat{X}_{s}$ under $\widehat{\mathbb{P}}_{x}$ we obtain

$$
\begin{aligned}
J & =\lim _{\epsilon \downarrow 0} \int_{0}^{1} \int_{E} \exp \left\{-\frac{\gamma}{m((y-\epsilon, y+\epsilon))} 1_{(y-\epsilon, y+\epsilon)}(z)\right\} g_{x}^{z}(s) m(d z) d s \\
& \leq \lim _{\epsilon \downarrow 0} \int_{0}^{1} \int_{E} g_{x}^{z}(s) m(d z) d s=\int_{0}^{1} \widehat{\mathbb{P}}_{x}(R>s) d s,
\end{aligned}
$$

which is finite due to Lemma 5.1.

We are ready to formulate the main result of this section.

Theorem 5.3. For fixed $\gamma>0, x, y \in E$ and $t>0$ define $\widehat{l}_{x, y, \gamma}(t):=\widehat{\mathbb{E}}_{x} e^{-\gamma \widehat{L}_{t}(y)}$ and $l_{x, y, \gamma}(v):=\mathbb{E}_{x} e^{-\gamma L_{v}^{y}}$. Assume that for every $\lambda>0$

$$
\int_{0}^{\infty} e^{-\lambda t} \widehat{l}_{x, y, \gamma}(t) d t<\infty
$$

If $\bar{\mu}_{x, y, \gamma}^{l}$ is the measure defined by

$$
\bar{\mu}_{x, y, \gamma}^{l}([0, s)):=\mathbb{E}_{x} \int_{0}^{s} e^{-\gamma L_{v}^{y}} d L_{v}^{x}, \quad s \geq 0
$$

then

$$
\widehat{l}_{x, y, \gamma} * \bar{\mu}_{x, y, \gamma}^{l}=1 * l_{x, y, \gamma} .
$$

In particular, if $\widehat{l}_{x, x, \gamma}(t):=\lim _{y \downarrow} \widehat{l}_{x, y, \gamma}(t)$ and (5.3) holds for $\widehat{l}_{x, x, \gamma}(t)$ then

$$
\left(1-l_{x, x, \gamma}\right) * \widehat{l}_{x, x, \gamma}=\gamma * l_{x, x, \gamma}
$$

Proof: Notice that $\widehat{l}_{x, y, \gamma}(t)$ is well defined for $t>0$ due to Lemma 5.2. The proof is based on counting excursions. Let $\lambda, \gamma, t>0$. Define

$$
\begin{gathered}
I(t):=\mathbb{E}_{x} \sum_{\tau_{s-<t}} e^{-\lambda \tau_{s-}-\gamma L_{\tau_{s-}}^{y}}\left(\int_{0}^{\sigma_{x}} e^{-\lambda v-\gamma L_{v}^{y}} d s\right) \circ \theta_{\tau_{s-}}, \\
I I(t):=\mathbb{E}_{x} \int_{t}^{d_{t}} e^{-\lambda v-\gamma L_{v}^{y}} d v, \quad I I I(t):=\mathbb{E}_{x} \int_{0}^{t} e^{-\lambda v-\gamma L_{v}^{y}} d v .
\end{gathered}
$$

Again, by the master formula of excursions

$$
I(t)=\left(\mathbb{E}_{x} \int_{0}^{t} e^{-\lambda v-\gamma L_{v}^{y}} d L_{v}^{x}\right) \int_{U} F_{\lambda, \gamma}(u) \widehat{\mathbb{P}}_{x}(d u),
$$


where $F_{\lambda, \gamma}(u)=\int_{0}^{R(u)} e^{-\lambda v-\gamma \widehat{L}_{v}(y)} d v$. For $v \leq t$ we defined $\widehat{l}_{x, y, \gamma}(v)=\widehat{\mathbb{E}}_{x} e^{-\gamma \widehat{L}_{v}(y)}$. Clearly $\int_{U} F_{\lambda, \gamma}(u) \widehat{\mathbb{P}}_{x}(d u)=\int_{0}^{\infty} e^{-\lambda v} \widehat{l}_{x, y, \gamma}(v) d v$. For any $t>0$ we defined a $\sigma$-finite measure $\bar{\mu}_{x, y, \gamma}^{l}$ on $[0, t)$ by (5.4), so by Fubini's theorem

$$
\mathbb{E}_{x} \int_{0}^{t} e^{-\lambda v-\gamma L_{v}^{y}} d L_{v}^{x}=\int_{0}^{t} e^{-\lambda v} \bar{\mu}_{x, y, \gamma}^{l}(d v)
$$

Letting $t \rightarrow \infty$ and putting just written equalities together yield

$$
\lim _{t \rightarrow \infty} I(t)=\left[\int_{0}^{\infty} e^{-\lambda v} \bar{\mu}_{x, y, \gamma}^{l}(d v)\right]\left[\int_{0}^{\infty} e^{-\lambda v} \widehat{l}_{x, y, \gamma}(v) d v\right],
$$

and the last is finite by assumption on the second term.

To compute $I I(t)$ we write by Markov property

$$
I I(t)=\mathbb{E}_{x} e^{-\lambda t-\gamma L_{t}^{y}} \mathbb{E}_{X_{t}} \int_{0}^{\sigma_{x}} e^{-\lambda s-\gamma L_{s}^{y}} d s .
$$

Observe that

$$
\mathbb{E}_{X_{t}} \int_{0}^{\sigma_{x}} e^{-\lambda s-\gamma L_{s}^{y}} d s \leq \frac{1}{\lambda}\left(1-\mathbb{E}_{X_{t}} e^{-\lambda \sigma_{x}}\right),
$$

so we clearly see that $\lim _{t \rightarrow \infty} I I(t)=0$. Finally we observe that for $l_{x, y, \gamma}(v)=$ $\mathbb{E}_{x} e^{-\gamma L_{v}^{y}}$ we have by Fubini's theorem

$$
\lim _{t \rightarrow \infty} \operatorname{III}(t)=\int_{0}^{\infty} e^{-\lambda v} l_{x, y, \gamma}(v) d v .
$$

Since $I(t)-I I(t)=I I I(t)$, we conclude that

$$
\left[\int_{0}^{\infty} e^{-\lambda v} \bar{\mu}_{x, y, \gamma}^{l}(d v)\right]\left[\int_{0}^{\infty} e^{-\lambda v} \widehat{l}_{x, y, \gamma}(v) d v\right]=\frac{1}{\lambda} \int_{0}^{\infty} e^{-\lambda v} l_{\gamma}(v) d v
$$

and by Laplace transform argument

$$
\widehat{l}_{x, y, \gamma} * \bar{\mu}_{x, y, \gamma}^{l}=1 * l_{x, y, \gamma} .
$$

By the proof of Lemma $5.2 y \mapsto \widehat{l}_{x, y, \gamma}(t)$ is bounded and $\widehat{l}_{x, x, \gamma}(t):=\lim _{y \downarrow x} \widehat{l}_{x, y, \gamma}(t)$ is well defined. By a standard calculus

$$
\mathbb{E}_{x} e^{-\lambda t-\gamma L_{t}^{x}}=1-\lambda \mathbb{E}_{x} \int_{0}^{t} e^{-\lambda v-\gamma L_{v}^{x}} d v-\gamma \mathbb{E}_{x} \int_{0}^{t} e^{-\lambda v} e^{-\gamma L_{v}^{x}} d L_{v}^{x}
$$

and since $l_{x, x, \gamma}(v)=\mathbb{E}_{x} e^{-\gamma L_{v}^{x}}$ we obtain

$$
\mathbb{E}_{x} \int_{0}^{t} e^{-\lambda v-\gamma L_{v}^{x}} d L_{v}^{x}=\frac{1}{\gamma}\left[1-\mathbb{E}_{x} e^{-\lambda t-\gamma L_{t}^{x}}-\lambda \int_{0}^{t} e^{-\lambda v} l_{x, x, \gamma}(v) d v\right] .
$$

By assumption, (5.3) holds, so we have

$$
\lim _{t \rightarrow \infty} I(t)=\frac{1}{\gamma}\left[1-\lambda \int_{0}^{\infty} e^{-\lambda v} l_{x, x, \gamma}(v) d v\right]\left[\int_{0}^{\infty} e^{-\lambda v} \widehat{l}_{x, x, \gamma}(v) d v\right] .
$$

Hence, comparing $I(t)-I I(t)$ and $I I I(t)$ with $t \rightarrow \infty$ yields

$$
\frac{1}{\gamma}\left[\int_{0}^{\infty} e^{-\lambda v}\left(1-l_{x, x, \gamma}(v)\right) d v\right]\left[\int_{0}^{\infty} e^{-\lambda v} \widehat{l}_{x, x, \gamma}(v) d v\right]=\frac{1}{\lambda} \int_{0}^{\infty} e^{-\lambda v} l_{x, x, \gamma}(v) d v .
$$


Finally, by Laplace transform argument

$$
\left(1-l_{x, x, \gamma}\right) *\left(\frac{1}{\gamma} \widehat{l}_{x, x, \gamma}\right)=1 * l_{x, x, \gamma}
$$

Example 5.4. We will give some examples of using Theorem 5.3 in case $x=y$. For $\gamma>0$ we have

$$
\left(1-l_{x, x, \gamma}\right) * \widehat{l}_{x, x, \gamma}=\gamma * l_{x, x, \gamma},
$$

which is the Volterra integral equation of the first kind with respect to $\widehat{l}_{x, x, \gamma}$. As can be seen below, even in typical cases, equation (5.7) is quite complicated.

If $X$ is a Brownian motion and $x=0$ then the distribution of $L_{t}^{0}$ is well known and

$$
l_{0,0, \gamma}(t)=e^{\frac{\gamma^{2} t}{2}}\left(1-\operatorname{Erf}\left(\gamma \sqrt{\frac{t}{2}}\right)\right), t \geq 0
$$

where Erf denotes the error function $\operatorname{Erf}(z)=\frac{2}{\sqrt{\pi}} \int_{0}^{z} e^{-u^{2}} d u$. Equation (5.7) takes the form

$$
\begin{aligned}
& \int_{0}^{t}\left(1-e^{\frac{\gamma^{2}(t-u)}{2}}\left(1-\operatorname{Erf}\left(\gamma \sqrt{\frac{t-u}{2}}\right)\right)\right) \widehat{l}_{0,0, \gamma}(u) d u \\
& =\gamma \int_{0}^{t} e^{\frac{\gamma^{2} u}{2}}\left(1-\operatorname{Erf}\left(\gamma \sqrt{\frac{u}{2}}\right)\right) d u,
\end{aligned}
$$

which may be solved either by numerical methods (see Linz, 1969) or by the techniques of deconvolution presented in Jakubowski and Wiśniewolski (2020).

The formula for $l_{x, x, \gamma}$ in case of $X$ being a squared Bessel process with negative index $\mu \in(-1,0)$ and $x=0$, is given in Jakubowski and Wiśniewolski $(2020+$, Ex. 5.4)

$$
l_{0,0, \gamma}(t)=E_{-\mu}\left(-\gamma \kappa(\mu) \Gamma(-\mu) t^{-\mu}\right),
$$

where $E_{a}$ is the Mittag-Leffler function with parameter $a$ and $\kappa(\mu):=$ $\left(2^{\mu} \Gamma(1+\mu)\right)^{-1}$. Equation (5.7) takes the form

$$
\begin{aligned}
& \int_{0}^{t}\left(1-E_{-\mu}\left(-\gamma \kappa(\mu) \Gamma(-\mu)(t-u)^{-\mu}\right)\right) \widehat{l}_{0,0, \gamma}(u) d u \\
& =\gamma \int_{0}^{t} E_{-\mu}\left(-\gamma \kappa(\mu) \Gamma(-\mu) u^{-\mu}\right) d u .
\end{aligned}
$$

To formulate the next results we need some background on convolutions of measures. Recall that by $\mathcal{A}$ we denote the algebra of convolutions of locally integrable functions on a non-negative half-line.

Definition 5.5. We say that a signed measure $\mu^{\dagger}$ is a quasi-inverse of the signed measure $\mu$ if for any $f \in \mathcal{A}$

$$
f * \mu * \mu^{\dagger}=f * \mu+f * \mu^{\dagger}
$$


Proposition 5.6. For each $\sigma$-finite signed measure $\mu$ on $\mathcal{B}\left(\mathbb{R}_{+}\right)$such that $\mu(\{0\})<$ 1 there exists an unique quasi-inverse signed measure $\mu^{\dagger}$. Moreover

$$
-1 * \mu^{\dagger}=\sum_{n \geq 1} 1 * \mu^{* n}
$$

Before the proof of Proposition 5.6 we separate out an useful lemma.

Lemma 5.7. Assume that $\mu, \mu_{1}, \mu_{2}$ are $\sigma$-finite signed measures on $\mathcal{B}\left(\mathbb{R}_{+}\right)$and $\mu=\mu_{1}+\mu_{2}$. Assume that $\mu_{1}^{\dagger}$ exists and $\kappa:=\mu_{2}-\mu_{2} * \mu_{1}^{\dagger}$. If $\kappa^{\dagger}$ exists, then $\mu^{\dagger}$ exists and

$$
1 * \mu^{\dagger}=1 *\left(k^{\dagger}+\mu_{1}^{\dagger}-k^{\dagger} * \mu_{1}^{\dagger}\right)
$$

Proof: We follow the ideas of Lew (1972) for $\mathcal{A}$. It is well known that signed measures on $\mathcal{B}\left(\mathbb{R}_{+}\right)$constitute Banach algebra with convolution operation. Moreover, if $\delta$ denotes the neutral element of unitization of $\mathcal{A}$ then for each $\sigma$-finite signed measure $\mu_{1}$, for which $\mu_{1}^{\dagger}$ exists, the element $1 *\left(\delta-\mu_{1}\right)$ has an associated uniquely determined element $1 *\left(\delta-\mu_{1}\right)^{-1}$ which satisfies

$$
1 * 1 *\left(\delta-\mu_{1}\right) *\left(\delta-\mu_{1}\right)^{-1}=1 * 1
$$

or simply

$$
1 *\left(\delta-\mu_{1}\right) *\left(\delta-\mu_{1}\right)^{-1}=1 .
$$

Indeed, $\left(\delta-\mu_{1}\right)^{-1}$ is equal to $\delta-\mu_{1}^{\dagger}$, since one may easily check that

$$
1 *\left(\delta-\mu_{1}\right) *\left(\delta-\mu_{1}^{\dagger}\right)=1 .
$$

Moreover, if $\mu_{1}^{\dagger}$ and $\mu_{2}^{\dagger}$ exist then

$$
1 *\left(\delta-\mu_{1}\right)^{-1} *\left(\delta-\mu_{2}\right)^{-1}=1 *\left(\delta-\mu_{1}^{\dagger}\right) *\left(\delta-\mu_{2}^{\dagger}\right) .
$$

So if $\mu=\mu_{1}+\mu_{2}$, and we may define $\kappa:=\mu_{2}-\mu_{2} * \mu_{1}^{\dagger}$, then we observe that $\mu_{2}=\kappa *\left(\delta-\mu_{1}\right)$. Observe further that

$$
\begin{aligned}
1 *\left(\kappa^{\dagger}+\mu_{1}^{\dagger}-\kappa^{\dagger} * \mu_{1}^{\dagger}\right) & =-1 *\left(\delta-\mu_{1}^{\dagger}\right) *\left(\delta-\kappa^{\dagger}\right)+1 \\
& =-1 *\left(\delta-\mu_{1}\right)^{-1} *(\delta-\kappa)^{-1}+1 \\
& =-1 *\left(\delta-\mu_{1}-\kappa *\left(\delta-\mu_{1}\right)\right)^{-1}+1=-1 *(\delta-\mu)^{-1}+1,
\end{aligned}
$$

so it follows that $\mu^{\dagger}:=k^{\dagger}+\mu_{1}^{\dagger}-k^{\dagger} * \mu_{1}^{\dagger}$ is the desired quasi-inverse element.

Lemma 5.8. If $\mu$ is a $\sigma$-finite measure, then

$$
1 * \mu * \mu^{\dagger}=1 * \mu+1 * \mu^{\dagger} \quad \Leftrightarrow \quad \forall f \in \mathcal{A} \quad f * \mu * \mu^{\dagger}=f * \mu+f * \mu^{\dagger} .
$$

Proof: Follows by multiplying the LHS in sense of convolution by $f$ and the RHS by 1 respectively, and by the fact that $\mathcal{A}$ has no 0-divisors.

Proof: (of Proposition 5.6) We adopt the proof of Lemma 1 in Lew (1972) to the setup of $\sigma$-finite measures. By Lemma 5.7, for a signed measure $\mu$ it is enough to find decomposition $\mu=\mu_{1}+\mu_{2}$ such that $\mu_{1}^{\dagger}$ exists and for $\kappa:=\mu_{2}-\mu_{2} * \mu_{1}^{\dagger}$ the element $\kappa^{\dagger}$ exists as well. Not loosing generality assume that $\mu$ is a $\sigma$-finite measure. Due to hypothesis of the theorem we may choose a strictly positive $a$ such that $(1 * \mu)(a)<1$. Set $\mu_{1}:=\mu(\cdot \cap[0, a))$. Clearly $\sum_{n \geq 1} 1 * \mu_{1}^{* n}$ converges in $\mathcal{A}$, so we may define $-\mu_{1}^{\dagger}$ as a measure induced by the last sum or simply by 
an identity $1 *\left(-\mu_{1}^{\dagger}\right)=\sum_{n \geq 1} 1 * \mu_{1}^{* n}$. If we set $\mu_{2}=\mu-\mu_{1}$, then $\mu_{2}$ vanishes on any Borel subset of $[0, a)$ and so by the definition of convolution of measures $\mu_{2}^{* n}$ vanishes on any Borel subset of $[0, n a)$ and so does $\kappa^{* n}$ for $\kappa=\mu_{2}-\mu_{2} * \mu_{1}^{\dagger}$. As a result we may define $-\mu_{2}^{\dagger}$ and $-\kappa^{\dagger}$ as measures induced by $\sum_{n \geq 1} 1 * \mu_{2}^{* n}$ and $\sum_{n \geq 1} 1 * \kappa^{* n}$ respectively, or simply by identities $1 *\left(-\mu_{2}^{\dagger}\right)=\sum_{n \geq 1} 1 * \mu_{2}^{* n}$ and $1 *\left(-\kappa^{\dagger}\right)=\sum_{n \geq 1} 1 * \kappa^{* n}$.

One more lemma will be important in the sequel.

Lemma 5.9. Assume that $y, f \in \mathcal{A}$ and $\mu$ is a $\sigma$-finite measure on non-negative half-line. If $y=f+y * \mu$, then $y=f-f * \mu^{\dagger}$.

Proof: From assumption $y$ solves a Volterra integral equation of the second kind with respect to $\sigma$-finite measure. If there exists $w \in \mathcal{A}$ such that $w=f+w * \mu$ then $w=y$. Indeed, we would then have

$$
w * y=f * y+y * w * \mu=f * w+y * w * \mu,
$$

so the assertion about uniqueness follows from the fact that $\mathcal{A}$ has no 0-divisors. To conclude the proof we directly check that $y=f-f * \mu^{\dagger}$ satisfies the integral equation. Indeed,

$$
f+y * \mu=f+\left(f-f * \mu^{\dagger}\right) * \mu=f+f * \mu-f * \mu^{\dagger} * \mu=f-f * \mu^{\dagger}=y,
$$

where we used Lemma 5.8.

We use the notion of convolution of measures to describe the excursion local time. For this recall that for $l_{x, x, \gamma}(v)=\mathbb{E}_{x} e^{-\gamma L_{v}^{x}}$ we proved under assumptions of Theorem 5.3 that $\left(1-l_{x, x, \gamma}\right) * \widehat{l}_{x, x, \gamma}=\gamma * l_{x, x, \gamma}$.

Proposition 5.10. Fix $\gamma>0$. Assume that (5.3) holds for $y=x$. Assume that there exist a signed $\sigma$-finite measure $\widehat{\mu}$ and a function $\alpha \in \mathcal{A}$ on $[0, \infty)$ such that $\alpha(0)=-1$ and such that $l_{x, x, \gamma}$ has the following representation

$$
l_{x, x, \gamma}=(1+\alpha) * \widehat{\mu}-\alpha .
$$

Then

$$
(1+\alpha) * \widehat{l}_{x, x, \gamma}=\gamma * l_{x, x, \gamma}+\gamma * l_{x, x, \gamma} * \sum_{n \geq 1} \widehat{\mu}^{* n} .
$$

Proof: For $\alpha$ and $\widehat{\mu}$ as in the hypothesis of the theorem, the LHS of formula (5.6) may be rewritten in the following form

$$
\begin{aligned}
\left(1-l_{x, x, \gamma}\right) * \widehat{l}_{x, x, \gamma} & =1 * \widehat{l}_{x, x, \gamma}-\widehat{l}_{x, x, \gamma} *((1+\alpha) * \widehat{\mu}-\alpha) \\
& =(1+\alpha) * \widehat{l}_{x, x, \gamma}-(1+\alpha) * \widehat{l}_{x, x, \gamma} * \widehat{\mu},
\end{aligned}
$$

and comparing the last with RHS of (5.6) yields

$$
(1+\alpha) * \widehat{l}_{x, x, \gamma}-(1+\alpha) * \widehat{l}_{x, x, \gamma} * \widehat{\mu}=\gamma * l_{x, x, \gamma} .
$$

The last is integral equation with respect to $(1+\alpha) * \widehat{l}_{x, x, \gamma}$ as in Lemma 5.9 , so

$$
\begin{aligned}
(1+\alpha) * \widehat{l}_{x, x, \gamma} & =\gamma * l_{x, x, \gamma}-\gamma * l_{x, x, \gamma} *(\widehat{\mu})^{\dagger} \\
& =\gamma * l_{x, x, \gamma}+\gamma * l_{x, x, \gamma} * \sum_{n \geq 1} \widehat{\mu}^{* n},
\end{aligned}
$$

where in the last equality we used Proposition 5.6. 
Below we present some possible choices of $\alpha$ and $\widehat{\mu}$ for decomposition (5.8). We assume that assumptions of Theorem 5.3 are satisfied. The first example is theoretic.

Example 5.11. For $\lambda>0$ set $\alpha(t):=-e^{-\lambda t}$ and assume that $t \mapsto l_{x, x, \gamma}(t)$ is differentiable on $(0, \infty)$. Define $\Delta_{\lambda, \gamma}(t):=e^{\lambda t} l_{x, x, \gamma}(t)$. Then decomposition (5.8) holds, if

$$
\widehat{\mu}([0, t))=\frac{1}{\lambda} e^{-\lambda t} \frac{\partial \Delta_{\lambda, \gamma}(t)}{\partial t}, \quad t>0,
$$

defines a signed $\sigma$-finite measure. Indeed, taking $\alpha(t):=-e^{-\lambda t}$ equality (5.8) may be rewritten as

$$
l_{x, x, \gamma}(t)=\widehat{\mu}([0, t))-\int_{0}^{t} e^{-\lambda(t-u)} \widehat{\mu}(d u)+e^{-\lambda t},
$$

So

$$
\Delta_{\lambda, \gamma}(t)=e^{\lambda t} \widehat{\mu}([0, t))-\int_{0}^{t} e^{\lambda u} \widehat{\mu}(d u)+1
$$

As a result

$$
\frac{\partial \Delta_{\lambda, \gamma}(t)}{\partial t}=\lambda e^{\lambda t} \widehat{\mu}([0, t))
$$

which due to assumptions on $\Delta_{\lambda, \gamma}$ makes $\widehat{\mu}$ well defined.

The second example is constructive. The measure $\widehat{\mu}$ is chosen upfront.

Proposition 5.12. Let $\widehat{\mu}$ be the Lebesgue measure and $j(t):=t$. Then $\alpha$ defined by

$$
\alpha:=j-l_{x, x, \gamma}+\left(j-l_{x, x, \gamma}\right) * \sum_{n \geq 1} \widehat{\mu}^{* n},
$$

satisfies the hypothesis of Proposition 5.10.

Proof: Assuming that $\widehat{\mu}$ is given, we rewrite condition (5.8) as an equation with respect to $\alpha$. Hence

$$
\alpha=1 * \widehat{\mu}-l_{x, x, \gamma}+\alpha * \widehat{\mu}=j-l_{x, x, \gamma}+\alpha * \widehat{\mu},
$$

so to obtain (5.11) we use Lemma 5.9.

Example 5.13. Since $j-l_{x, x, \gamma}$ is locally integrable and $\widehat{\mu}$ is the Lebesgue measure, we have for any $t \geq 0$

$$
\alpha(t)=t-l_{x, x, \gamma}(t)+\sum_{n=1}^{\infty} \int_{0}^{t}\left(t-u-l_{x, x, \gamma}(t-u)\right) \frac{u^{n}}{n !} d u .
$$

For Itô-McKean diffusions the form of $l_{x, x, \gamma}$ is given explicitly in terms of the transition density:

$$
l_{x, x, \gamma}(t)=1+1 * \sum_{n=1}^{\infty}(-\gamma)^{n} p^{* n}(x, x)(t) .
$$

As a result, the terms of the RHS of (5.12) are given explicitly and give the form of the Volterra integral equation (5.9) for $\widehat{l}_{x, x, \gamma}$. 


\section{Non-linear excursion formula}

The master formula of excursion although surprisingly universal and useful for the theory, is actually a linear a transformation of some appropriately measurable process (depending on previsible or optional setup). The naturally raised question is about non-linear versions of the formula. Actually (to best of our knowledge) the known non-linear version is the exponential one (see Revuz and Yor, 1991, Ch. XII, Prop. 1.12). In this section we present a new non-linear version of excursion formula for additive functionals. The structure of the new formula reminds the structure of the linear master formula, where the Markovian kernel from the linear formula is replaced by a more complicated but still a Markovian counterpart.

Let us introduce some auxiliary notation. For a continuous additive $\mathbb{R}_{+}-$valued functional $\left(A_{t}\right)$ associated with Itô-McKean diffusion $X$, define

$$
i_{t}:=A_{\sigma_{x}} \circ \theta_{t}, \quad t \geq 0 .
$$

By some standard arguments one may conclude that $i_{\tau_{s-}^{x}}$ for $\tau_{s-}^{x}=\inf \left\{v>0: L_{v}^{x} \geq\right.$ $t\}$ is well defined and previsible (left-continuous). Recall that by $d_{t}^{x}$ we denote the first hitting time of $x$ by the process $X$, after $t$.

Definition 6.1. (Hypothesis $A$ ) We say that $A$ satisfies hypothesis $A$ if

(1) there exists a density with respect to Lebesgue measure, $\bar{g}$ of $A_{\sigma_{x}}$, that is

$$
\mathbb{P}_{w}\left(A_{\sigma_{x}} \in d z\right)=\bar{g}(w, z) d z,
$$

and for any fixed $z$ the map $w \mapsto \bar{g}(w, z)$ is measurable.

(2) there exists a kernel $\phi_{t, y}$ representing the distribution of the pair $\left(A_{t}, A_{d_{t}^{x}}\right)$ on condition $\left\{X_{t}=y\right\}$ :

$$
\mathbb{P}_{x}\left(A_{t} \in d v, A_{d_{t}^{x}} \in d k \mid X_{t}=y\right)=\phi_{t, y}(d v, k) d k,
$$

and $(t, y) \mapsto \phi_{t, y}(d v, k)$ is measurable for any fixed non-negative $k$.

We are ready to formulate the result of this section, namely the non-linear excursions formula for non-negative, continuous additive functionals of $X$.

Theorem 6.2. (Non-linear excursion formula) Let $t>0, x \in E$ be fixed and $h$ be a Borel, positive or bounded function on $\mathbb{R}_{+}$. Under hypothesis $A$ there exist a kernel $\mathcal{Q}_{s}^{t, x}$ such that

$$
\mathbb{E}_{x} h\left(\sum_{\tau_{s-}<t} i_{\tau_{s-}^{x}}\right)=\mathbb{E}_{x} \int_{0}^{t} \int_{0}^{\infty} h(k) \mathcal{Q}_{t-u}^{t, x}(d k) d L_{u}^{x} .
$$

The kernel is defined by

$$
\mathcal{Q}_{s}^{t, x}(d k)=\widehat{\mathbb{E}}_{x} \Psi^{t, x}\left(\widehat{X}_{s}, d k\right), \quad \Psi^{t, x}(y, d k)=\mathbb{P}_{x}\left(A_{d_{t}^{x}} \in d k \mid X_{t}=y\right) .
$$

Moreover, for $z \in E$ define a kernel $\overline{\mathcal{Q}}_{s}^{t, x, z}(d k)=\mathbb{E}_{z} \Psi^{t, x}\left(\widehat{X}_{s}^{(x)}, d k\right)$ where $\widehat{X}^{(x)}$ denotes the canonical process killed at first hitting of $x$ (not be confused with excursion process), and by $\mu_{s}$ denote the entrance law of excursion process $\widehat{X}$, that is $\mu_{s}(d k)=\widehat{\mathbb{P}}_{x}\left(\widehat{X}_{s} \in d k\right)$. Then the kernel $\left(\mathcal{Q}_{s}^{t, x}\right)$ is Markovian in the following sense: for any $u, s>0$ we have $\mathcal{Q}_{s+u}^{t, x}=\mu_{s} \overline{\mathcal{Q}}_{u}^{t, x, \cdot}$, which formally means

$$
\mathcal{Q}_{s+u}^{t, x}(d k)=\int_{E} \overline{\mathcal{Q}}_{u}^{t, x, z}(d k) \mu_{s}(d z)
$$


Proof: It is quite clear that $\sum_{\tau_{s-}^{x}<t} i_{\tau_{s-}^{x}}=i_{t}+A_{t}$, so for a chosen $h$ Borel, positive or bounded. Denote $I(t)=\sum_{\tau_{s-}^{x}<t} i_{\tau_{s-}^{x}}$. We have by Markov property

$$
\begin{aligned}
\mathbb{E}_{x} h(I(t)) & =\mathbb{E}_{x} h\left(A_{t}+A_{\sigma_{x}} \circ \theta_{t}\right)=\mathbb{E}_{x} \int_{0}^{\infty} h\left(A_{t}+z\right) \bar{g}\left(X_{t}, z\right) d z \\
& =\int_{0}^{\infty} \int_{0}^{\infty} \int_{E} h(v+z) \bar{g}(y, z) \mathbb{P}_{x}\left(X_{t} \in d y, A_{t} \in d v\right) d z \\
& =\int_{0}^{\infty} h(k) \int_{0}^{k} \bar{g}(y, k-v) \mathbb{P}_{x}\left(X_{t} \in d y, A_{t} \in d v\right) d k
\end{aligned}
$$

Recall that by occupation time formula for local time we have $\mathbb{E}_{x} L_{t}^{x}=\int_{0}^{t} p_{u}(x, x) d u$, and since $p(x, y)=p(y, x)$ and $p(y, x)=p(x, x) * g_{x}^{y}$ we obtain

$$
p_{t}(x, y)=\mathbb{E}_{x} \int_{0}^{t} g_{x}^{y}(t-u) d L_{u}^{x}
$$

As a result, by Fubini's theorem

$$
\begin{aligned}
& \mathbb{E}_{x} h(I(t)) \\
& =\mathbb{E}_{x} \int_{0}^{t} \int_{0}^{\infty} h(k)\left[\int_{E} \int_{0}^{k} \bar{g}(y, k-v) \mathbb{P}_{x}\left(A_{t} \in d v \mid X_{t}=y\right) g_{x}^{y}(t-u) m(d y)\right] d k d L_{u}^{x}
\end{aligned}
$$

Clearly

$$
\int_{0}^{k} \bar{g}(y, k-v) \mathbb{P}_{x}\left(A_{t} \in d v \mid X_{t}=y\right)=\int_{0}^{k} \mathbb{E}_{x}\left[1_{\left\{A_{t} \in d v\right\}} \bar{g}\left(X_{t}, k-v\right) \mid X_{t}=y\right] .
$$

By Markov property

$$
\bar{g}\left(X_{t}, z\right) d z=\mathbb{E}_{x}\left[A_{\sigma_{x}} \circ \theta_{t} \in d z \mid \mathcal{F}_{t}\right] .
$$

By hypothesis A

$$
\mathbb{P}_{x}\left(A_{t} \in d v, A_{d_{t}^{x}} \in d k \mid X_{t}=y\right)=\phi_{t, y}(d v, k) d k,
$$

so recalling that $A_{d_{t}^{x}}=A_{t}+A_{\sigma_{x}} \circ \theta_{t}$ we have

$$
\begin{aligned}
{\left[\int_{0}^{k} \bar{g}(y, k-v) \mathbb{P}_{x}\left(A_{t} \in d v \mid X_{t}=y\right)\right] d k } & =\left[\int_{0}^{k} \phi_{t, y}(d v, k)\right] d k \\
& =\mathbb{P}_{x}\left(A_{t} \leq k, A_{d_{t}^{x}} \in d k \mid X_{t}=y\right) .
\end{aligned}
$$

By the definition of $d_{t}^{x}$ the last probability is equal to $\mathbb{P}_{x}\left(A_{d_{t}^{x}} \in d k \mid X_{t}=y\right)$ which is exactly what we denote by $\Psi^{t, x}(y, d k)$. Thus, the first integrand in the square bracket of (6.5) may be replaced by $\Psi^{t, x}(y, d k)$, and we obtain

$$
\mathbb{E}_{x} h(I(t))=\mathbb{E}_{x} \int_{0}^{t} \int_{0}^{\infty} h(k)\left[\int_{E} \Psi^{t, x}(y, d k) g_{x}^{y}(t-u) m(d y)\right] d k d L_{u}^{x} .
$$

It was recalled several times in this paper that $g_{x}^{y}(u) m(d y)=\widehat{\mathbb{P}}_{x}\left(\widehat{X}_{u} \in d y\right)$, so

$$
\begin{aligned}
\mathbb{E}_{x} h(I(t)) & =\mathbb{E}_{x} \int_{0}^{t} \int_{0}^{\infty} h(k) \widehat{\mathbb{E}}_{x} \Psi^{t, x}\left(\widehat{X}_{t-u}, d k\right) d k d L_{u}^{x} \\
& =\mathbb{E}_{x} \int_{0}^{t} \int_{0}^{\infty} h(k) \mathcal{Q}_{t-u}^{t, x}(d k) d L_{u}^{x} .
\end{aligned}
$$

Since we are in the diffusive setup the kernel $\mathcal{Q}_{u}^{t, x}$ is measurable directly from the definition. To see that it is Markovian in the above specific sense we recall that if 
$\mu_{s}$ is the entrance law of excursion process and $\widehat{\mathcal{P}}_{u}^{(x)}$ denotes the semigroup of the process arising from $X$ and killed at $x$, we have $\mu_{u+s}=\mu_{u} \widehat{\mathcal{P}}_{s}^{(x)}$ for any positive $s$ and $u$. So we write

$$
\begin{aligned}
\mathcal{Q}_{s+u}^{t, x}(d k) & =\widehat{\mathbb{E}}_{x} \Psi^{t, x}\left(\widehat{X}_{s+u}, d k\right)=\int_{E} \Psi^{t, x}(z, d k) \mu_{s+u}(d z) \\
& =\int_{E} \int_{E} \Psi^{t, x}(z, d k) \widehat{p}_{s}^{(x)}(y, z) m(d z) \mu_{u}(d y) \\
& =\int_{E} \mu_{u}(d y)\left(\int_{E} \Psi^{t, x}(z, d k) \widehat{p}_{s}^{(x)}(y, z) m(d z)\right) \\
& =\int_{E} \mu_{u}(d y) \overline{\mathcal{Q}}_{s}^{t, x, y}(d k) .
\end{aligned}
$$

This finishes the proof.

Example 6.3. For Itô-McKean diffusion $X$ and $x=0$ recall the setup of Salminen et al. (2007). Take $A_{t}=t$. It follows from formula Salminen et al. (2007, (6)) that hypothesis A is satisfied. Moreover,

$$
\begin{aligned}
\mathbb{P}_{0}\left(X_{t} \in d y, d_{t}^{0} \in d k\right) & =\left(\int_{0}^{t} p_{u}(0,0) g_{0}^{y}(t-u) d u\right) g_{0}^{y}(k-t) d k m(d y) \\
& =p_{t}(y, 0) g_{0}^{y}(k-t) d k m(d y)
\end{aligned}
$$

so that

$$
\Psi^{t, 0}(y, d k)=g_{0}^{y}(k-t) d k
$$

We obtain

$$
\mathcal{Q}_{s}^{t, 0}(d k)=\left(\int_{E} g_{0}^{y}(k-t) g_{0}^{y}(s) m(d y)\right) d k
$$

\section{References}

Alili, L., Patie, P., and Pedersen, J. L. Representations of the first hitting time density of an Ornstein-Uhlenbeck process. Stoch. Models, 21 (4), 967-980 (2005). MR2179308.

Blumenthal, R. M. Excursions of Markov processes. Probability and its Applications. Birkhäuser Boston, Inc., Boston, MA (1992). ISBN 0-8176-3575-0. MR1138461.

Bogus, K. and Małecki, J. Sharp estimates of transition probability density for Bessel process in half-line. Potential Anal., 43 (1), 1-22 (2015). MR3361787.

Borodin, A. N. and Salminen, P. Handbook of Brownian motion-facts and formulae. Probability and its Applications. Birkhäuser Verlag, Basel, second edition (2002). ISBN 3-7643-6705-9. MR1912205.

Byczkowski, T., Jakubowski, J., and Wiśniewolski, M. On functionals of excursions for Bessel processes with negative index. Studia Math., 246 (3), 217-231 (2019). MR3883303.

Friedman, A. and Shinbrot, M. Volterra integral equations in Banach space. Trans. Amer. Math. Soc., 126, 131-179 (1967). MR206754.

Getoor, R. K. Excursions of a Markov process. Ann. Probab., 7 (2), 244-266 (1979). MR525052. 
Gripenberg, G., Londen, S.-O., and Staffans, O. Volterra integral and functional equations, volume 34 of Encyclopedia of Mathematics and its Applications. Cambridge University Press, Cambridge (1990). ISBN 0-521-37289-5. MR1050319.

Hamana, Y. The probability distributions of the first hitting times of radial Ornstein-Uhlenbeck processes. Studia Math., 251 (1), 65-88 (2020). MR4042415.

Itô, K. Poisson point processes attached to Markov processes. In Proceedings of the Sixth Berkeley Symposium on Mathematical Statistics and Probability (Univ. California, Berkeley, Calif., 1970/1971), Vol. III: Probability theory, pp. 225-239 (1972). MR0402949.

Itô, K. and McKean, H. P., Jr. Diffusion processes and their sample paths. SpringerVerlag, Berlin-New York (1974). MR0345224.

Jakubowski, J. and Wiśniewolski, M. A convolution formula for the local time of an Itô diffusion reflecting at 0 and a generalized Stroock-Williams equation $(2020+)$. To appear in Bernoulli.

Jakubowski, J. and Wiśniewolski, M. Volterra integral equations of the first kind and applications to linear diffusions. Trans. Amer. Math. Soc., 373 (10), 74557472 (2020). MR4155213.

Kent, J. T. Eigenvalue expansions for diffusion hitting times. Z. Wahrsch. Verw. Gebiete, 52 (3), 309-319 (1980). MR576891.

Knight, F. B. Characterization of the Levy measures of inverse local times of gap diffusion. In Seminar on Stochastic Processes, 1981 (Evanston, Ill., 1981), volume 1 of Progr. Prob. Statist., pp. 53-78. Birkhäuser, Boston, Mass. (1981). MR647781.

Le Gall, J.-F. Brownian excursions, trees and measure-valued branching processes. Ann. Probab., 19 (4), 1399-1439 (1991). MR1127710.

Lew, J. S. On linear Volterra integral equations of convolution type. Proc. Amer. Math. Soc., 35, 450-456 (1972). MR308699.

Linz, P. Numerical methods for Volterra integral equations of the first kind. Comput. J., 12, 393-397 (1969). MR253577.

Lipton, A. and Kaushansky, V. On the First Hitting Time Density of an OrnsteinUhlenbeck Process. ArXiv Mathematics e-prints (2018). arXiv: 1810.02390.

Mijatović, A. and Pistorius, M. Continuously monitored barrier options under Markov processes. Math. Finance, 23 (1), 1-38 (2013). MR3015232.

Naĭmark, M. A. Normed rings. Translated from the first Russian edition by Leo F. Boron. P. Noordhoff N. V., Groningen (1959). MR0110956.

Revuz, D. and Yor, M. Continuous martingales and Brownian motion, volume 293 of Grundlehren der Mathematischen Wissenschaften [Fundamental Principles of Mathematical Sciences]. Springer-Verlag, Berlin (1991). ISBN 3-540-52167-4. MR1083357.

Rogers, L. C. G. and Williams, D. Diffusions, Markov processes, and martingales. Vol. 2. Itô calculus. Wiley Series in Probability and Mathematical Statistics: Probability and Mathematical Statistics. John Wiley \& Sons, Inc., New York (1987). ISBN 0-471-91482-7. MR921238.

Salminen, P., Vallois, P., and Yor, M. On the excursion theory for linear diffusions. Jpn. J. Math., 2 (1), 97-127 (2007). MR2295612.

Titchmarsh, E. C. The Zeros of Certain Integral Functions. Proc. London Math. Soc. (2), 25, 283-302 (1926). MR1575285. 
Trotter, H. F. A property of Brownian motion paths. Illinois J. Math., 2, 425-433 (1958). MR96311. 